\title{
Theories of change for e-health interventions targeting HIV/STIs and sexual risk, substance use and mental ill health amongst men who have sex with men: systematic review and synthesis
}

\author{
Rebecca Meiksin ${ }^{1 *}$ (D) G. J. Melendez-Torres², Jane Falconer ${ }^{1}$, T. Charles Witzel' ${ }^{1}$, Peter Weatherburn ${ }^{1}$ and
} Chris Bonell ${ }^{1}$

\begin{abstract}
Background: Sexual risk, substance use, and mental ill health constitute a syndemic of co-occurring, mutually reinforcing epidemics amongst men who have sex with men (MSM). Developed since 1995, e-health interventions offer accessible, anonymous support and can be effective in addressing these outcomes, suggesting the potential value of developing ehealth interventions that address these simultaneously amongst MSM. We conducted a systematic review of e-health interventions addressing one or more of these outcomes amongst MSM and in this paper describe the theories of change underpinning relevant interventions, what these offer and how they might complement each other.

Methods: We identified eligible reports via expert requests, reference-checking and database and Google searches. Results were screened for reports published in 1995 or later; focused on MSM; reporting on e-health interventions providing ongoing support to prevent HIV/STIS, sexual risk behaviour, substance use, anxiety or depression; and describing intervention theories of change. Reviewers assessed report quality, extracted intervention and theory of change data, and developed a novel method of synthesis using diagrammatic representations of theories of change.

Results: Thirty-three reports on 22 intervention theories of change were included, largely of low/medium-quality. Inductively grouping these theories according to their core constructs, we identified three distinct groupings of theorised pathways. In the largest, the 'cognitive/skills' grouping, interventions provide information and activities which are theorised to influence behaviour via motivation/intention and self-efficacy/perceived control. In the 'selfmonitoring' grouping, interventions are theorised to trigger reflection, self-reward/critique and self-regulation. In the 'cognitive therapy' grouping, the theory of change is rooted in cognitive therapy techniques, aiming to reframe negative emotions to improve mental health.

(Continued on next page)
\end{abstract}

\footnotetext{
* Correspondence: Rebecca.Meiksin@lshtm.ac.uk

'London School of Hygiene \& Tropical Medicine, 15-17 Tavistock Place, London WC1H 9SH, UK

Full list of author information is available at the end of the article
}

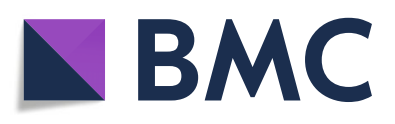

(- The Author(s). 2021 Open Access This article is licensed under a Creative Commons Attribution 4.0 International License, which permits use, sharing, adaptation, distribution and reproduction in any medium or format, as long as you give appropriate credit to the original author(s) and the source, provide a link to the Creative Commons licence, and indicate if changes were made. The images or other third party material in this article are included in the article's Creative Commons licence, unless indicated otherwise in a credit line to the material. If material is not included in the article's Creative Commons licence and your intended use is not permitted by statutory regulation or exceeds the permitted use, you will need to obtain permission directly from the copyright holder. To view a copy of this licence, visit http://creativecommons.org/licenses/by/4.0/. The Creative Commons Public Domain Dedication waiver (http://creativecommons.org/publicdomain/zero/1.0/) applies to the data made available in this article, unless otherwise stated in a credit line to the data. 
(Continued from previous page)

Conclusions: The synthesised theories of change provide a framework for developing e-health interventions that might holistically address syndemic health problems amongst MSM. Improving reporting on theories of change in primary studies of e-health interventions would enable a better understanding of how they are intended to work and the evidence supporting this. The novel diagrammatic method of theory of change synthesis used here could be used for future reviews where interventions are driven by existing well-defined behaviour and behaviour change theories.

Systematic review registration: PROSPERO CRD42018110317

Keywords: e-Health, Digital health, Men who have sex with men, Sexual health, HIV, STI, Substance use, Mental health, Systematic review, Theory of change

\section{Introduction}

Despite major advances in treatments and pharmacological prevention, men who have sex with men (MSM) continue to experience increased risk of infection with HIV and other sexually transmitted infections (STIs) [1] and high levels of sexual risk behaviour [2]. MSM also report high rates of alcohol [3-5] and legal and illegal drug use (henceforth referred to as substance use) [6-8] and of common markers of mental ill health [9]. MSM are twice as likely to be depressed or anxious compared to other men [10]. Nationally representative surveys in the UK suggest that almost half of MSM experience one or more of these outcomes [11].

Sexual risk, substance use and mental ill health are increasingly considered to constitute a syndemic of simultaneous, mutually reinforcing epidemics. There is consistent evidence that these outcomes can intercorrelate strongly at the level of the individual and the sexual event [12-18]. Drug use can be both a symptom and cause of mental ill health, and both drug use and mental ill health can increase sexual risk behaviours [19, 20]. MSM who report using certain drugs, such as nitrite inhalants, and ketamine and other drugs linked to sex parties, are more likely to report sexual risk with multiple partners [21]. Survey data indicate that MSM reporting substance use are more likely to report unprotected anal intercourse and HIV infection [22]; MSM with higher levels of anxiety and depression are more likely to have potential alcohol dependency [2]; and MSM with depressive symptoms report more condomless anal sex [23]. Public health strategies to address these outcomes together therefore have the potential to achieve multiplicative effects.

Stigma and discrimination pose barriers to appropriate services for MSM worldwide [24-26]. Online global surveys of MSM have found that only $11 \%$ of substanceusing participants reported accessible treatment programmes, with only $5 \%$ reporting use [27], and that fewer than half of participants had easy access to condoms and HIV testing [28]. A study of MSM attending UK sexual health clinics found that $42 \%$ of those with depressive symptoms were not diagnosed and $48 \%$ were not receiving treatment [29]. There is an urgent need for accessible and effective new strategies to address these outcomes amongst MSM.

e-Health interventions, facilitated via mobile phones, internet or other electronic communication technology, can be delivered in a variety of formats such as text messaging, smartphone/mobile apps, games and other online content. e-Health interventions aim to promote healthy behaviours and mental health, for example, by setting and reviewing goals; increasing/maintaining motivation; providing feedback on behaviour and challenging thought patterns that obstruct change. There is good evidence from systematic reviews focused on general or mixed populations that e-health interventions can reduce alcohol use [30] and address common causes of mental ill health [31-37]. Emerging evidence also suggests that e-health interventions might reduce drug use and sexual risk behaviour [38-41]. Given the clustered and interacting nature of these problems amongst MSM, this might suggest the value of developing e-health interventions for MSM that addresses these outcomes simultaneously and holistically. Such an approach might well have multiplicative effects. However, existing mental health [42] and HIV prevention [43] ehealth interventions rarely target MSM, and those that are not designed for MSM risk unacceptability or failure to address the specific needs of this population [42].

We therefore conducted a systematic review of ehealth interventions addressing sexual health, substance use and/or mental health amongst MSM to explore the approaches and theories of how the interventions were intended to work to improve targeted health outcomes ("theories of change") employed; factors promoting or impeding delivery or receipt of such interventions; their effectiveness in improving health outcomes and whether such interventions are cost-effective. We reviewed relevant interventions that targeted our three health outcomes of interest separately or together to maximise learning about what interventions exist that might be harnessed to address this syndemic and what the evidence from interventions addressing some but not all of these outcomes suggest could be developed to target all three holistically. 
In this paper, we report findings from our synthesis of intervention theories of change to understand how these interventions are intended to work. Intervention theories of change typically draw on existing scientific theories of behaviour (which consider factors that predict behaviours) and/or existing scientific theories of behaviour change (which propose general mechanisms of changing behaviour). Intervention theories of change make explicit the hypothesised mechanisms through which intervention activities are intended to generate outcomes [44], helping developers to systematically consider and describe which psychological, social or other factors interventions should address to achieve their intended outcomes. They also help evaluators determine what to measure to assess whether and how an intervention works and which components, if any, are most important [44]. Review evidence suggests that increased use of scientific theory is associated with greater effectiveness of internet-based health behaviour change interventions [45], but intervention theories of change must take into account which mechanisms sustain the targeted outcomes in a particular context, amongst a particular population [46]. Our aim is to describe the range of intervention theories of change that have informed e-health interventions, what these offers and how they might complement each other, which has not been done in previous reviews addressing our outcomes of interest [39, 41, 42, 47, 48]. We aim to answer the following questions:

1 What theories of change inform e-health interventions addressing HIV/STIs, sexual risk behaviour, substance use or common mental illness symptoms amongst MSM?

2 What overarching theories of change are suggested by the theories of change used for included interventions?

\section{Methods}

\section{Search strategy}

Our methods were guided by a protocol prospectively registered on PROSPERO in September 2018 (registration number CRD42018110317) [49].

In this paper, we report what intervention theories of change were used in eligible studies that were retrieved as part of a broader systematic review. Reports eligible for the broader review were published in 1995 or later (because e-health interventions were unavailable prior to this); focused on gay, bisexual and other men (including cisgender and transgender men) who have sex with men; and reported on e-health interventions delivered via mobile phone, internet or other electronic communication technology that aimed to provide ongoing support to populations consisting entirely or principally of MSM to prevent HIV, STIs, sexual risk behaviour, alcohol and drug use or common causes of mental ill health (anxiety or depression). We excluded e-health interventions merely facilitating one-off as opposed to ongoing support, those addressing HIV self-testing, clinic attendance or STI partner notification only, and interventions delivered by human providers via electronic media. There were no limitations by language or geographical location of studies. Reports eligible for inclusion in the theory of change synthesis described intervention theories of change, logic models or theorised mechanisms of effect.

Our search strategy, refined after piloting, covered two core concepts: men who have sex with men; and ehealth. Publication date was limited from 1995 to now and no language limits or limits by study methodology were added. We searched 19 health and social science bibliographic databases, 23 October-26 November 2018. These were ProQuest Applied Social Sciences Index \& Abstracts (ASSIA) (1987-current); Campbell Library (complete database); CRD Databases (complete databases); EBSCO CINAHL Plus (complete database); Wiley Cochrane Library (complete database); EPPI-Centre Database of Health Promotion Research (Bibliomap) (complete database); ProQuest Dissertations \& Theses Global (1951-current); OvidSP EconLit (1886 to October 18, 2018); OvidSP Embase (1980 to 2018 week 43); OvidSP Global Health (1910 to 2018 week 41); OvidSP Health Management Information Consortium (HMIC) (1979 to July 2018); ProQuest IBSS (1951-current); Ovid MEDLINE(R) and Epub Ahead of Print, In-Process \& Other Non-Indexed Citations and Daily (1946 to October 22, 2018); OvidSP PsycInfo (1806 to October 2018 week 3,); Web of Science Science Citation Index Expanded (1970-present, data last updated 24 October 2018); Elsevier Scopus (complete database); OvidSP Social Policy \& Practice (201807); Web of Science Social Sciences Citation Index Expanded (1970-present, data last updated 24. October 2018); ProQuest Sociological Abstracts (1952-current as of 29 October 2018). Full details of the search strings for each are available at the London School of Hygiene \& Tropical Medicine's Data Repository [50] and full details of the search conducted in the OvidSP Medline database are available in Additional file 1 . We also searched three clinical trials registers for relevant ongoing and unpublished studies (ClinicalTrials.gov; the World Health Organisation International Clinical Trials Registry Platform; the EPPICentre Trials Register of Promoting Health Interventions); Google for non-governmental organisation and governmental publications (limited to the first 100 results); the OpenGrey database; and references of included reports. We contacted experts to request eligible reports. Citations were uploaded to EndNote and deduplicated. Database and trials register searches were updated 22-27 April 2020. 


\section{Screening and data extraction}

Two reviewers piloted an inclusion criteria worksheet by screening batches of the same 50 references, resolving disagreements by consensus. After piloting achieved an agreement rate of at least 95\%, each reference was screened on title and abstract by one reviewer. A second round of screening with a comparable piloting process then focused on full study reports. Where reports provided insufficient information on the intervention to determine eligibility, we contacted study authors. Two reviewers independently extracted data from each eligible report on intervention theory of change constructs and mechanisms, the evidence presented in support of the theory of change and how the theory of change was developed. We extracted data on intervention theory of change as well as the existing scientific theories of behaviour and behaviour change that informed these.

\section{Quality assessment}

Two reviewers independently assessed the quality of each report included in the theory of change synthesis (i.e. each "theory report") using a tool used in our previous systematic reviews $[51,52]$, modified to include assessment of whether intervention mechanisms were theorised as operating differently by context [53]. Quality assessment focused on (a) the extent to which the theory of change described the path from intervention to outcomes; (b) the clarity with which theoretical constructs were defined; (c) the clarity with which causal inter-relationships between constructs were defined; (d) the extent to which the mechanisms underlying these inter-relationships were explained and (e) the extent to which the theory of change considered how mechanisms and resultant outcomes might vary by context. The two reviewers met to compare their assessments, resolving any differences through discussion. Rather than restricting the synthesis to studies judged to be of high quality, conclusions drawing on poorer quality reports were given less interpretive weight.

\section{Synthesis of intervention theories}

We undertook synthesis of author narratives describing theories of how interventions were intended to generate outcomes. We aimed first to summarise theories of change for specific interventions and then to examine whether there were one or more overarching theories of change relevant across different interventions. Theory synthesis commonly uses a meta-ethnographic approach, originally developed to synthesise findings across multiple qualitative studies [54], and now applied to theory synthesis. As originally applied to qualitative research, meta-ethnographic methods draw on primary constructs (verbatim qualitative data presented in reports of primary research) and secondary constructs (author interpretations of data presented in primary research) to develop tertiary constructs (reviewer interpretations presented in syntheses). Applied to theory synthesis, such methods draw solely on primary constructs (author descriptions of theory of change).

We initially planned to undertake line-by-line coding of theory reports in order to identify narrative themes within, and common themes across, intervention theories of change as we have done in previous theory syntheses $[51,52]$. In the first stage of our analysis two reviewers piloted this approach, using data extracted from the two highest-quality studies of similar interventions $[55,56]$. We applied line-by-line codes, beginning with in vivo codes which closely reflected the words used in the theory reports. We then grouped and organised codes, applying axial codes reflecting higher-order themes and met to compare and contrast the resulting coding. Because this approach did not readily capture the complex and well-described interrelationships between theoretical constructs present in the reports, we instead decided to develop a novel diagrammatic approach to theory synthesis. This methodological innovation allowed us to summarise the components of each intervention's theory of change and the explicit and/or implied causal relationships between them, drawing on text and diagrams present in the studies. Summarising these diagrammatically also facilitated comparison and synthesis of these components and relationships across included theories of change. Specifically, we drew diagrams of theories of change based on author text and diagrams, first for each intervention and then for overarching theories of change, which applied across multiple interventions. Like the approach we had initially planned, this novel method of theory synthesis was a form of qualitative synthesis, but one that aimed to describe theories of change primarily in terms of constructs, inter-connections and interactions rather than as narrative themes. Like conventional thematic analysis, it involved an initial stage of 'in vivo' coding of author descriptions to identify theories of change for each intervention (but expressed diagrammatically rather than as a set of narrative themes), followed by a stage of 'axial' coding to explore inter-connections between in vivo coding, identifying similarities and differences across interventions to develop overarching theories of change (again expressed diagrammatically).

Where more than one publication reported on the same intervention, reviewers drew on the theory of change descriptions from all relevant reports to inform the diagram. The two reviewers then met to compare the two diagrams for each intervention and reconcile discrepancies through discussion. Drawing on the strengths of each, we developed an overall diagram of each intervention's theory of change, which included intervention components, mediators and moderators (where 
discussed by authors) and intended outcomes. Where author descriptions implied but did not explicitly state interrelationships between components of the theory of change, reviewers made inferences and noted where the diagrams were in part based on such inferences.

Finding that the theory of change approaches underpinning the interventions were not patterned by targeted outcomes, we took an inductive approach, grouping diagrams of theories of change that shared important constructs. Then, using reciprocal translation (to identify and describe similar concepts occurring across theories of change underpinning different interventions), refutational synthesis (to identify contradictory or opposing concepts occurring across theories of change) and line of argument (to synthesise distinct elements occurring across theories of change that form part of a broader whole) approaches from meta-ethnography [57], each reviewer independently analysed the diagrams within each grouping. They did this by systematically examining the constructs and the relationships between constructs presented in each intervention-specific diagram and by examining whether they recurred, appeared only once or conflicted with those depicted in other interventionspecific diagrams within the grouping. Based on their analyses, each reviewer then independently drafted one synthesised diagram for each grouping of similar intervention theories of change.

We documented each stage of this process, noting where theory of change components or relationships between these components differed between individual diagrams within the grouping; the approach taken to synthesise these components (i.e. reciprocal translation, refutational synthesis, line of argument or the exclusion of a theory of change component); and the resulting decision for the synthesised theory of change diagram. The two reviewers then met to compare their respective synthesised diagrams for each grouping, reconciling discrepancies and drawing on the strengths of each to develop a single synthesised theory of change diagram for each theory of change grouping. To demonstrate this process, Additional file 2 presents the theory of change diagrams for each individual intervention in one grouping and the resulting diagram of the synthesised theory of change for that grouping. Each synthesised theory of change was given a descriptive title inductively drawing on the central approaches of the theories of change synthesised.

In this application of meta-ethnographic methods to the synthesis of theories of change, our first-order constructs were the theory of change information described in theory reports and represented in data extraction forms; our second-order constructs (analogous to in vivo codes) were the reviewers' interpretations of these concepts, represented in the intervention-specific theory of change diagrams and our third-order constructs (analogous to axial codes) were the higherorder interpretations, represented by the diagrams of the synthesised theories of change developed for each inductive grouping.

\section{Results}

\section{Screening}

Our search yielded 26,044 unique references, of which 37 were included in the overall review. Of these, 33 reported on intervention theories of change (see Fig. 1).

\section{Reports included in the intervention theory of change synthesis}

The 33 reports included in the theory of change synthesis represent 28 unique studies and 23 interventions [55, 56, 58-87]. Two interventions-a tailored and a non-tailored version of the Cognitive Vaccine Approach, both reported by Davidovich et al.-share a single theory of change [70], resulting in 22 unique theories of change included in the synthesis. Table 1 summarises each intervention theory of change; evidence supporting the theory of change; how the theory of change was developed and existing scientific theories on which it draws.

\section{Quality assessment}

Agreement between independent reviewer assessments was good, ranging from agreement on none of the five quality criteria $(N=1)$ to $5 / 5(N=15)$, with agreement of $4 / 5$ or above for more than three quarters of reports $(N=26 ; 79 \%)$. All disagreements on independent quality assessments were resolved by discussion. Table 2 shows quality assessment results for each theory report.

Quality varied notably across theory reports and clustered towards low and medium quality, with 14 reports (42\%) meeting none or one of the assessed criteria [61, 63-65, 76, 77, 79-82, 84, 86-88]; 14 (42\%) meeting two or three criteria $[56,58,59,62,66-68,71-75,78,83]$ and only five (15\%) meeting four or five criteria [55, 60, $69,70,85]$.

Quality varied across the five criteria assessed. Nearly three quarters of theory reports $(N=23 ; 70 \%)$ clearly defined constructs or concepts and about half described a pathway from intervention components to intended outcomes $(N=16 ; 48 \%)$. Slightly more than a third clearly described how theoretical constructs were inter-related $(N=12 ; 36 \%)$. Only four (12\%) described underlying mechanisms and the same number described how mechanisms and outcomes might vary by context.

\section{Scientific theories informing intervention design}

Authors cited a number of existing scientific theories informing intervention theories of change. Interventions often drew on more than one existing scientific theory. 


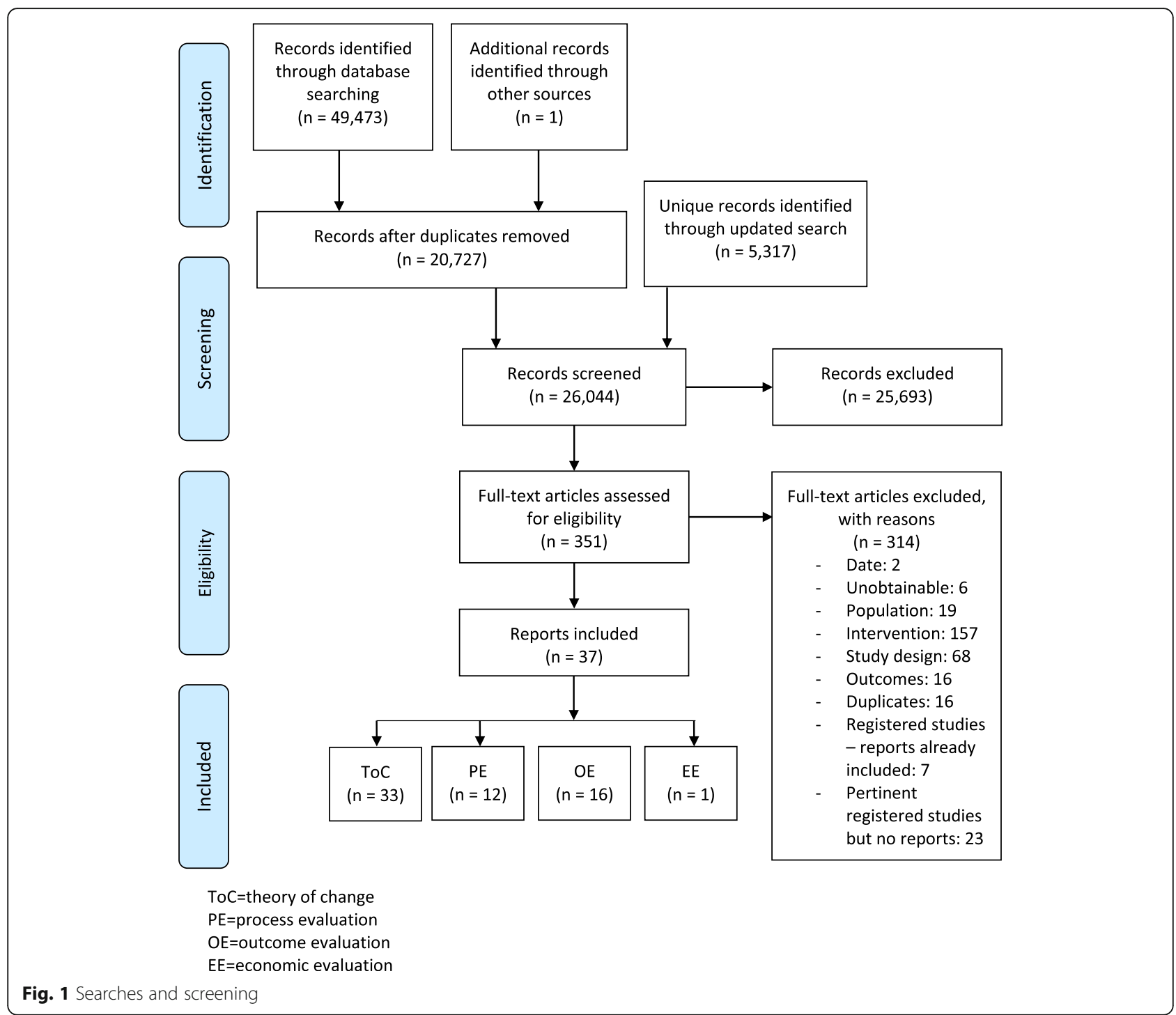

The information-motivation-behavioural skills (IMB) model and social cognitive theory were the most commonly cited, with the former informing seven interventions $[55,56,60,61,64,68,70,77-80,83,87]$ and the latter informing eight $[62,63,66,67,69,72,73,76,81$, 84]. The former was initially developed to inform HIV prevention but has since been advanced as a model for conceptualising individual and social determinants of health behaviours more broadly [90], and the latter incorporates individual, social and structural factors shaping, and techniques for changing, health behaviours [91]. One report citing social cognitive theory also cited its predecessor [92] social learning theory [72], and one report cited the social-personal framework which the authors describe as building on social learning theory [88]. The Smartphone Self-Monitoring intervention was underpinned by the theorised role of self-monitoring in supporting self-management [85], and we noted that self-monitoring is a core construct of social cognitive theory [89].

Other scientific theories of behaviour commonly used to inform health interventions informed between one and three interventions each: the health-belief model $[70,81]$, which focuses on the role of individual beliefs about health problems [93]; the theory of planned behaviour [59, 69, 70], which takes into account individual and social factors and theorises that intentions and perceptions of behavioural control are the direct precursors to behaviours [94] and social support theory [81], which theorises that support or perceptions of support from people who are trusted can reduce the stress of, and improve coping with, difficult events [95]. Content for the Sexpulse intervention was informed by the sexual health model, which identifies ten components essential to healthy sexuality [96], theorising that 'sexually healthy persons are more likely to make sexually healthy 


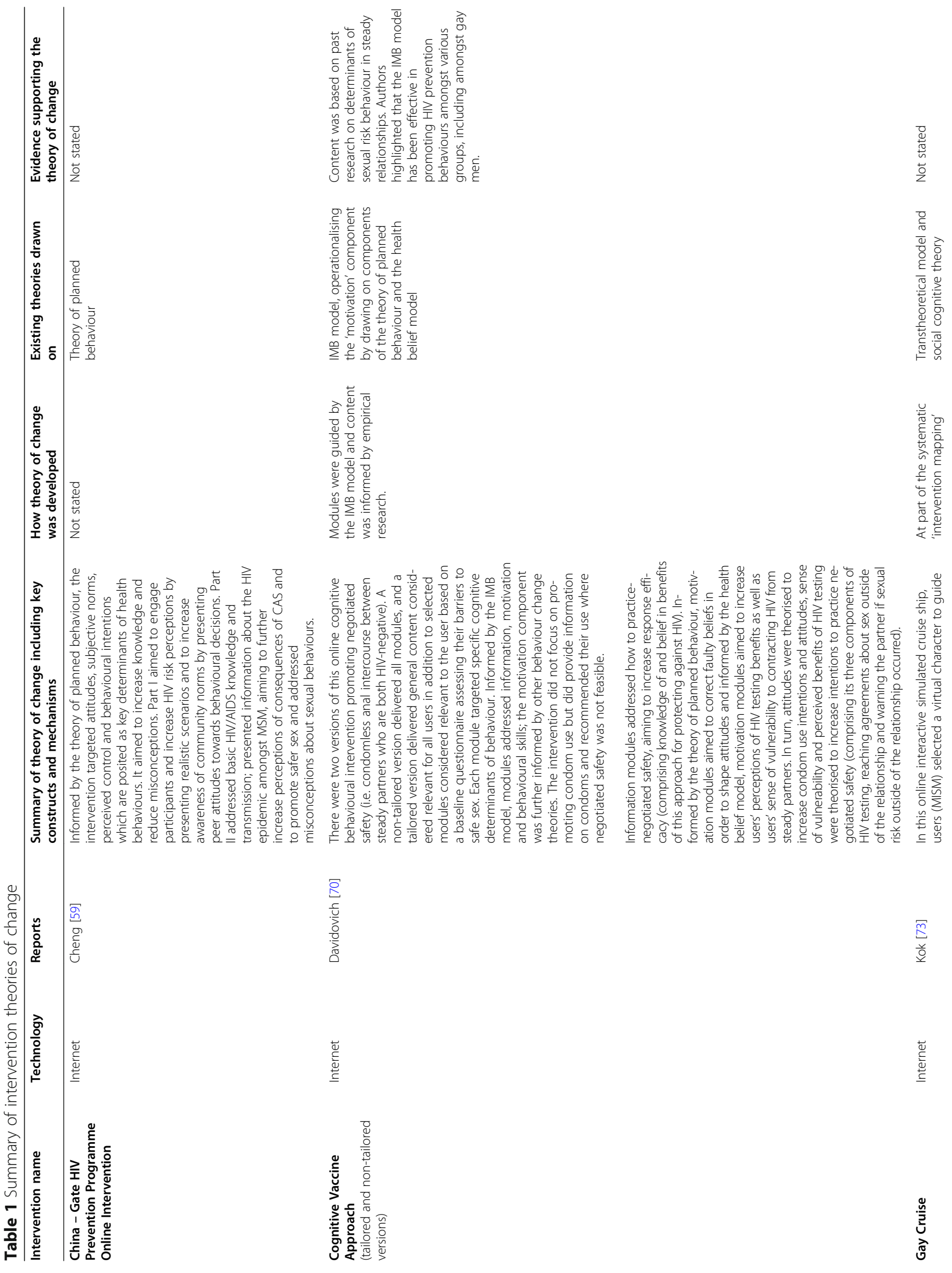



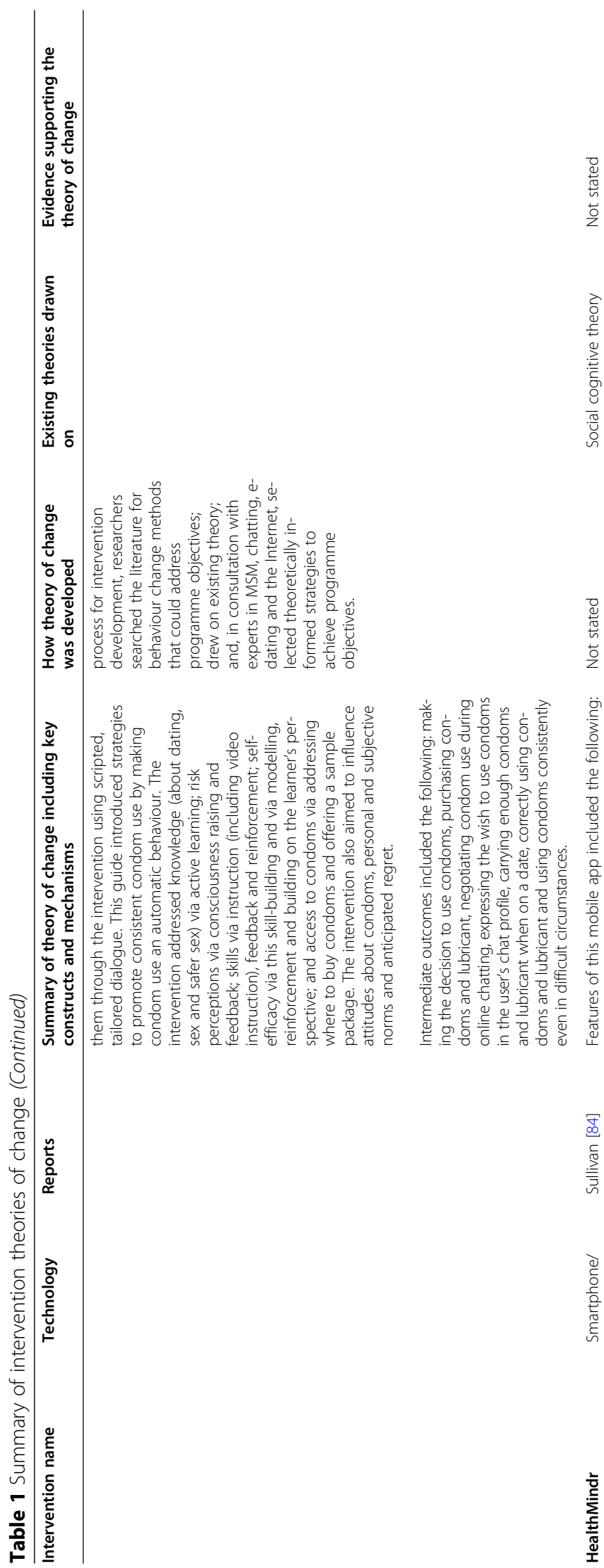

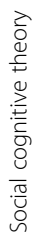

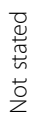

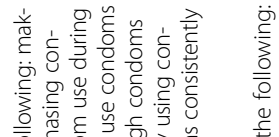

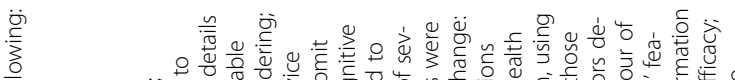

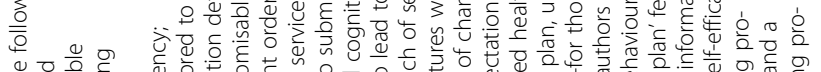

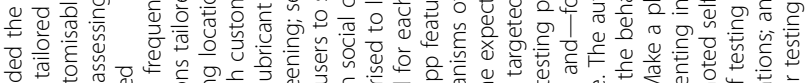

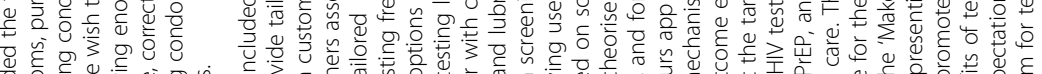

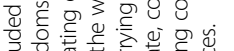

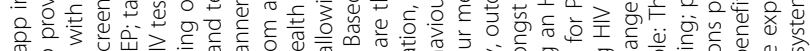

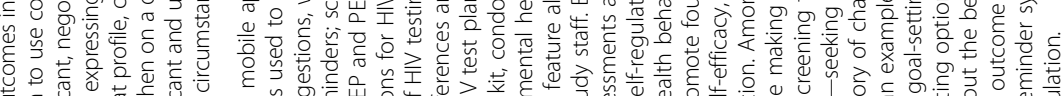

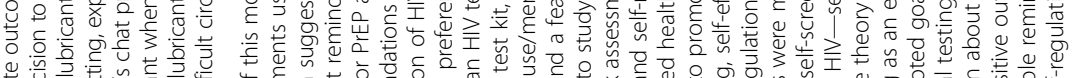

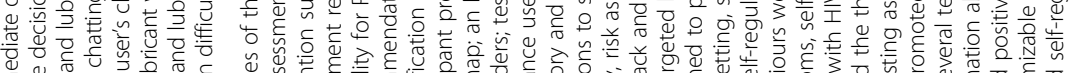

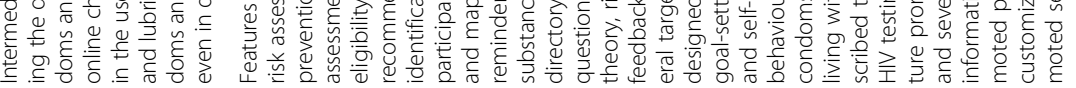

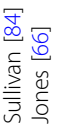

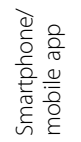




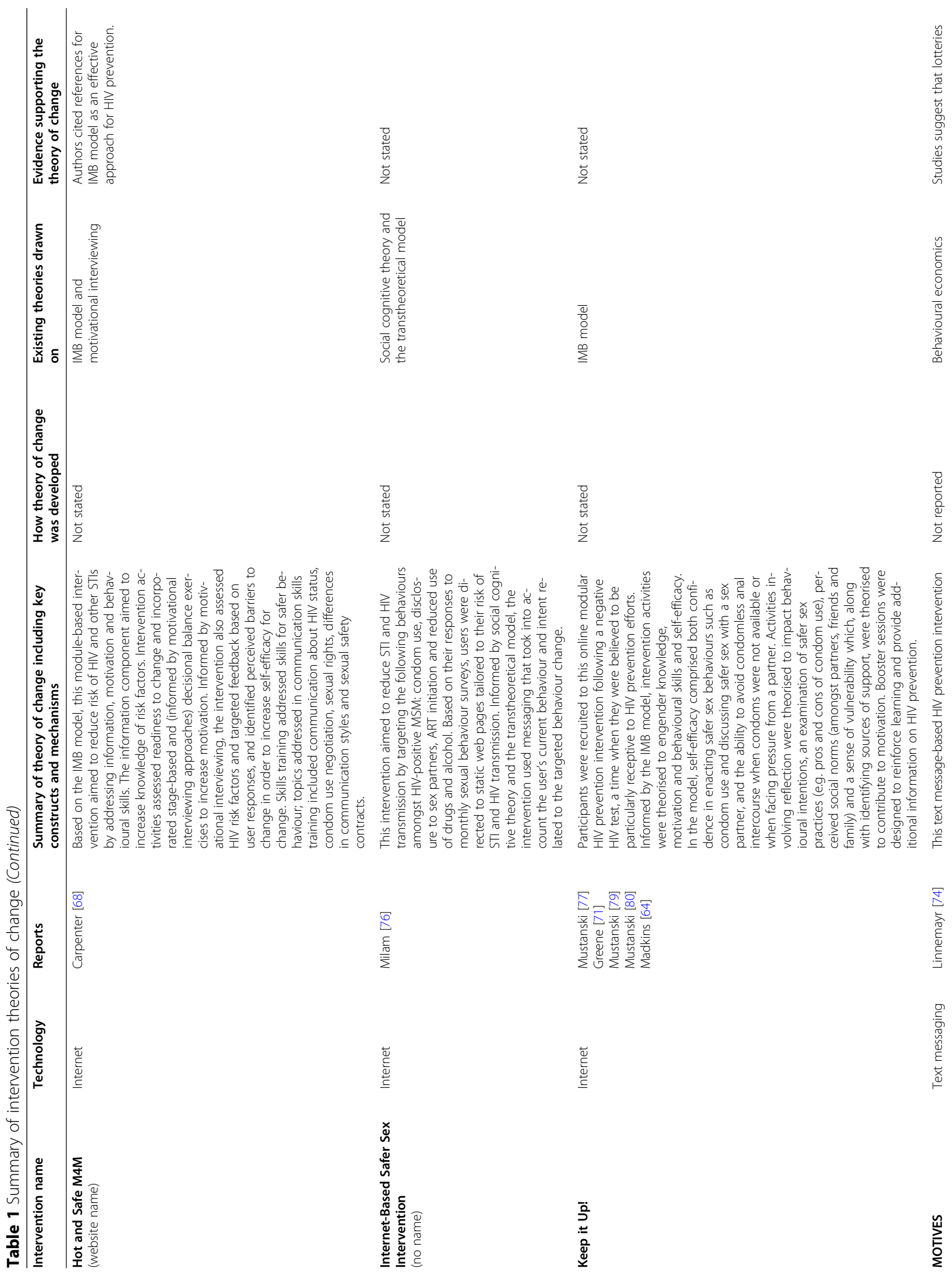



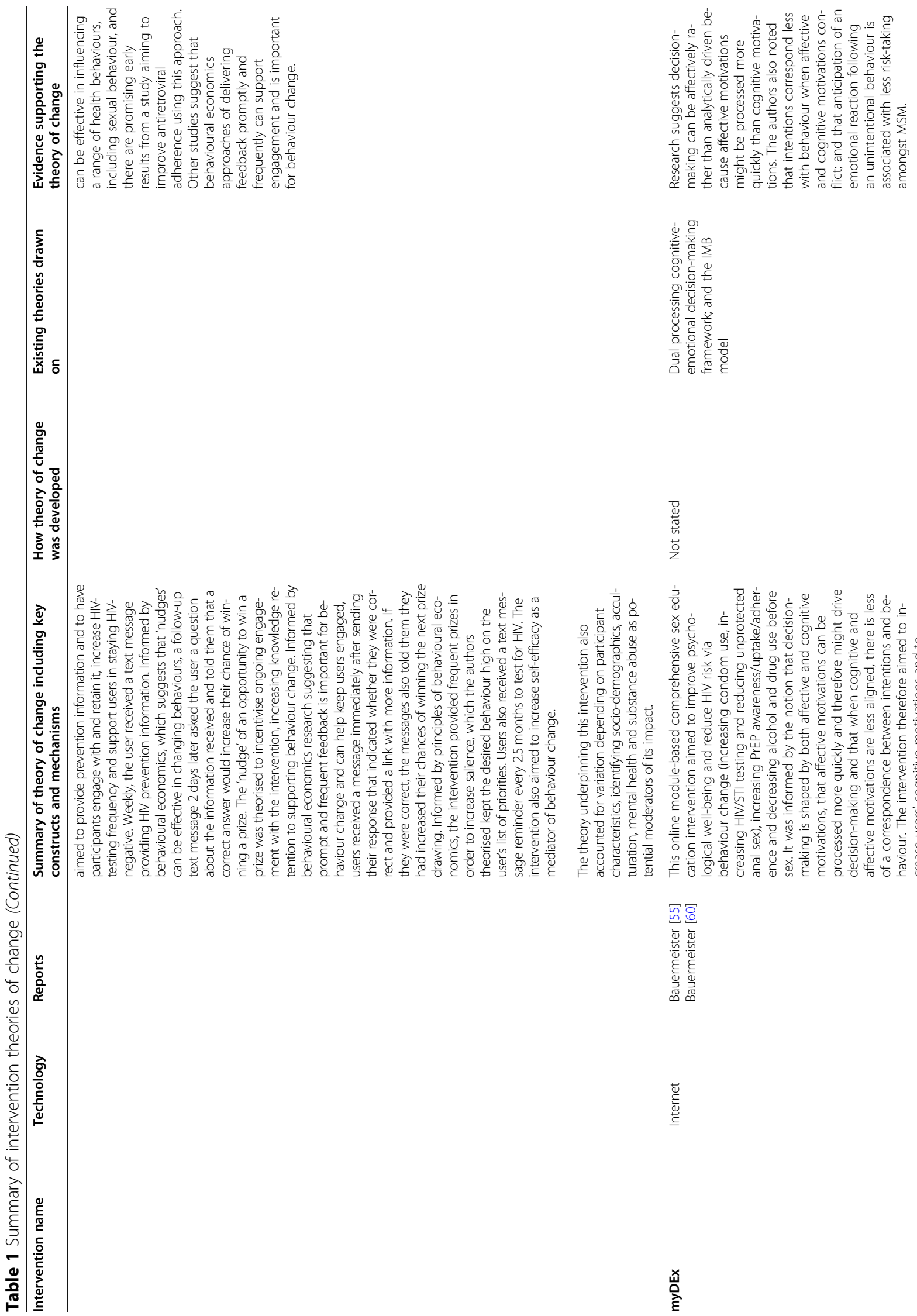

总

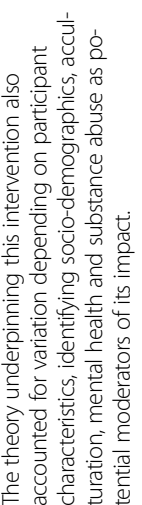
章

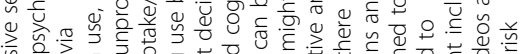

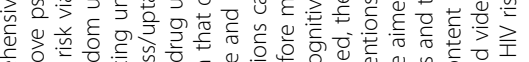

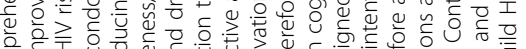

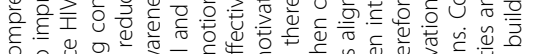

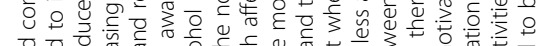

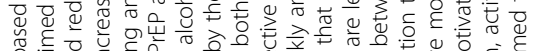

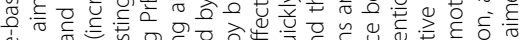

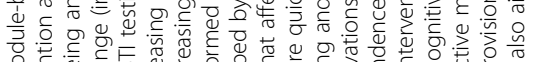

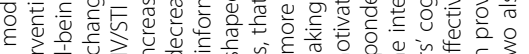

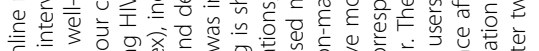

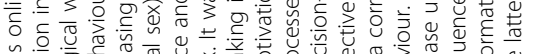

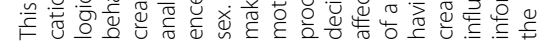
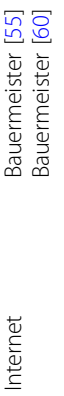

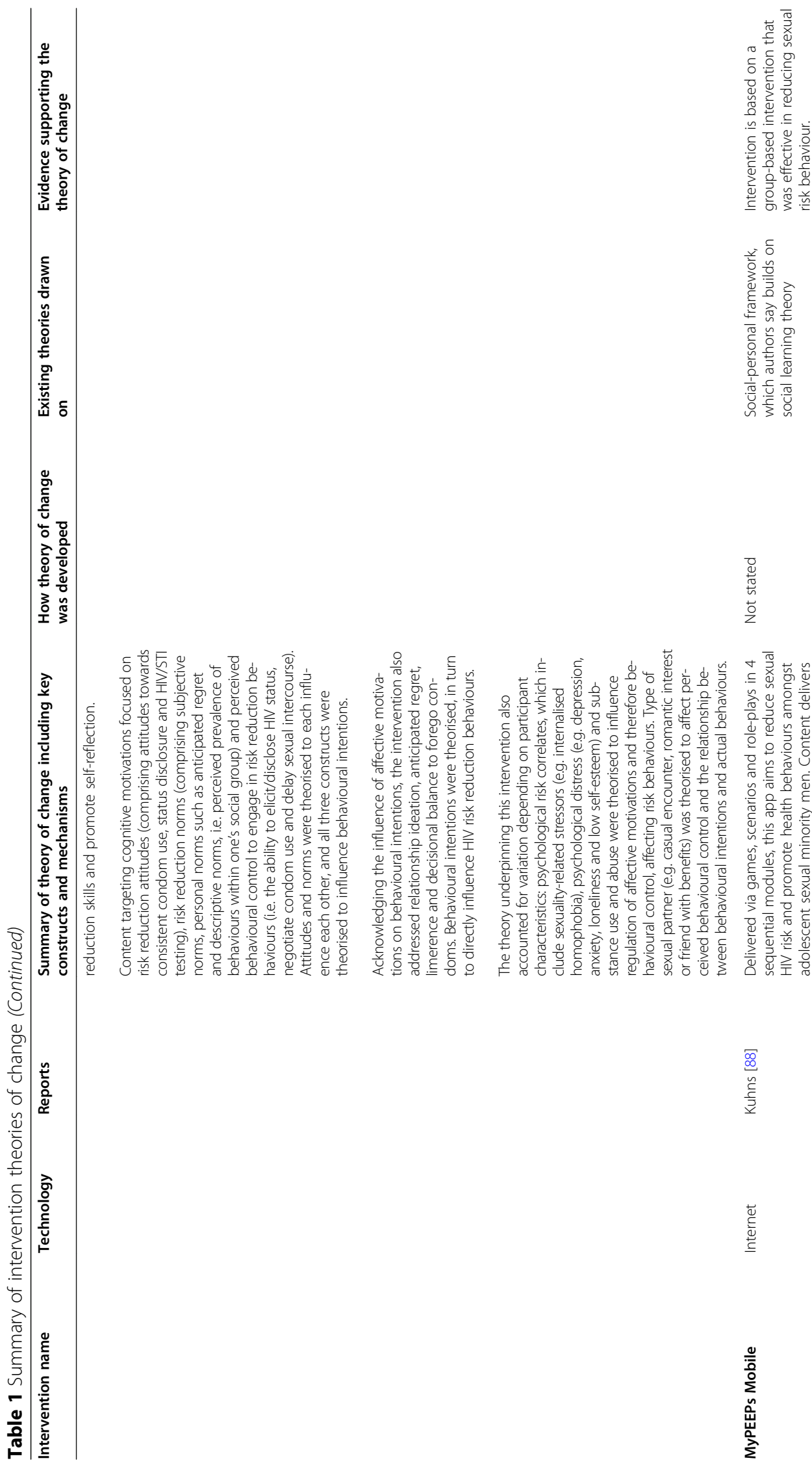

惫
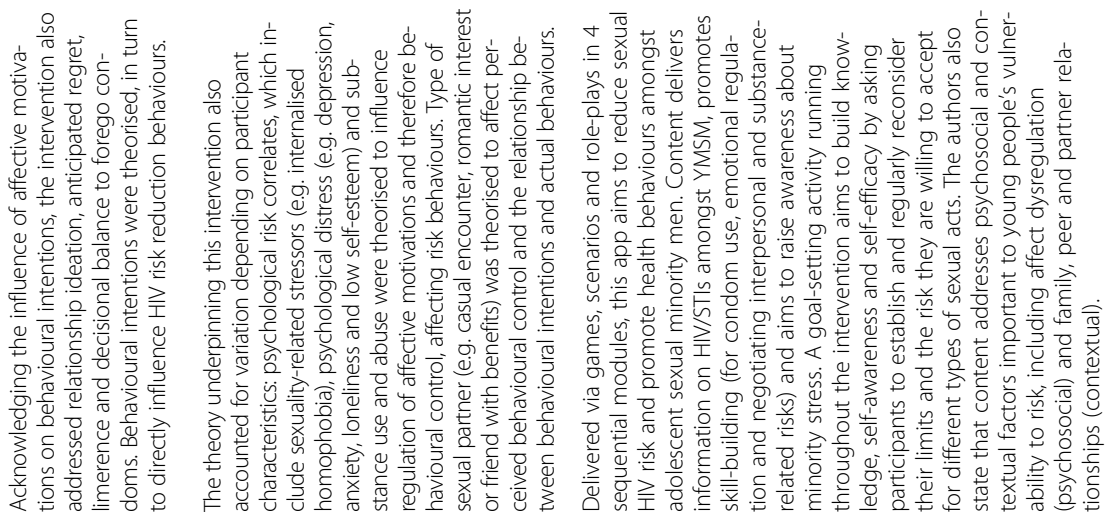

$\infty$
$\infty$
0

$\frac{1}{3}$
$\frac{1}{2}$

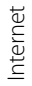

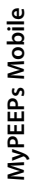




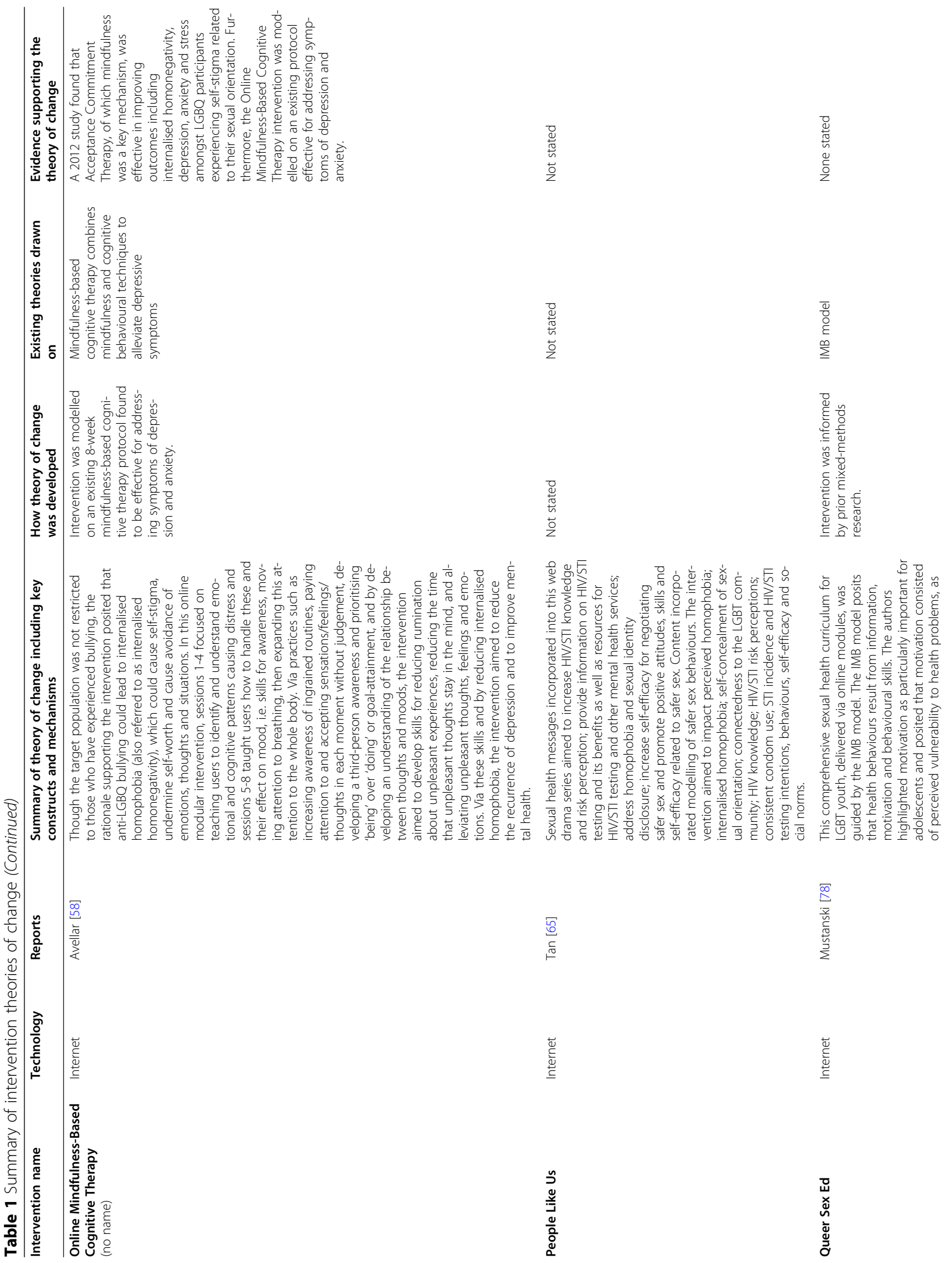




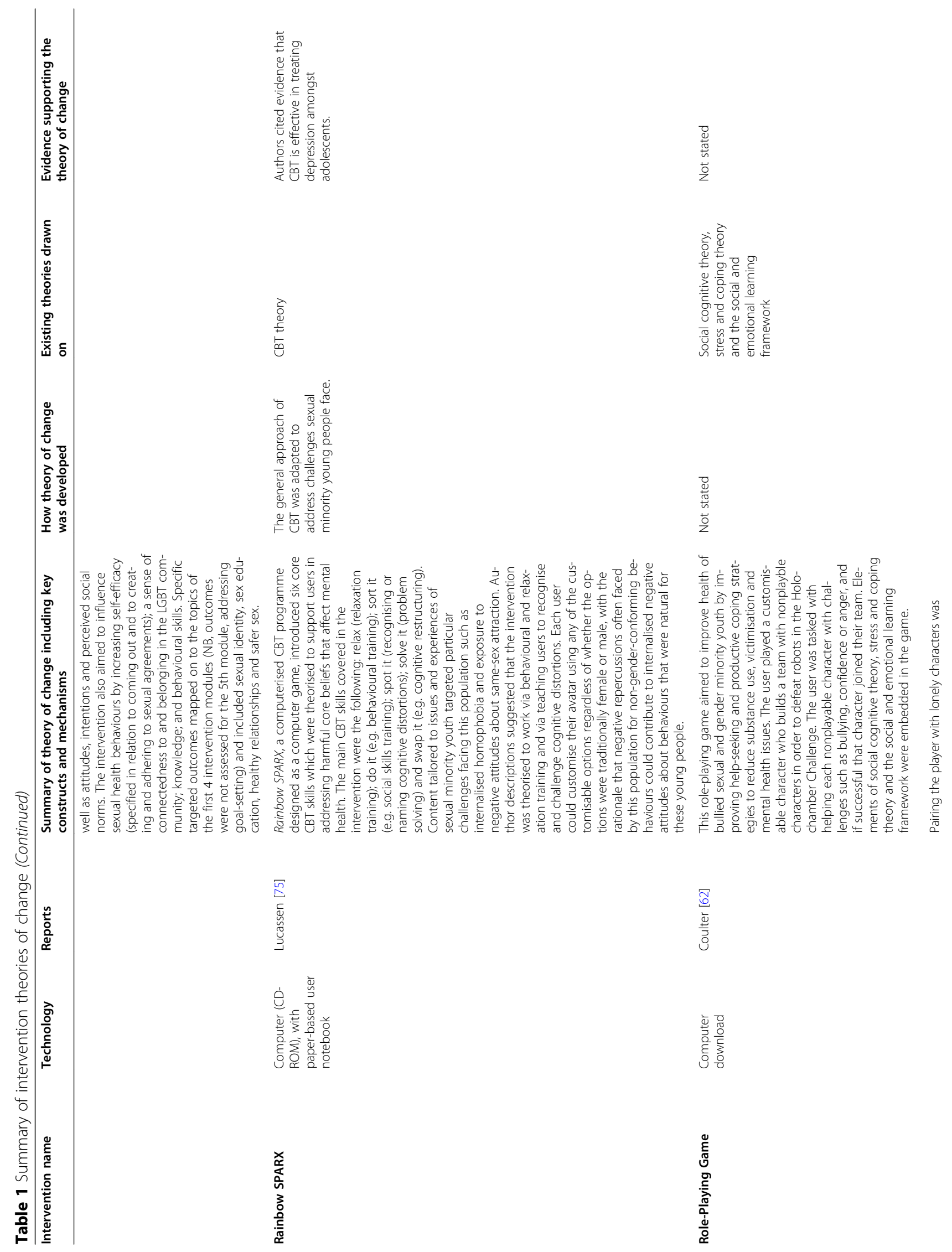




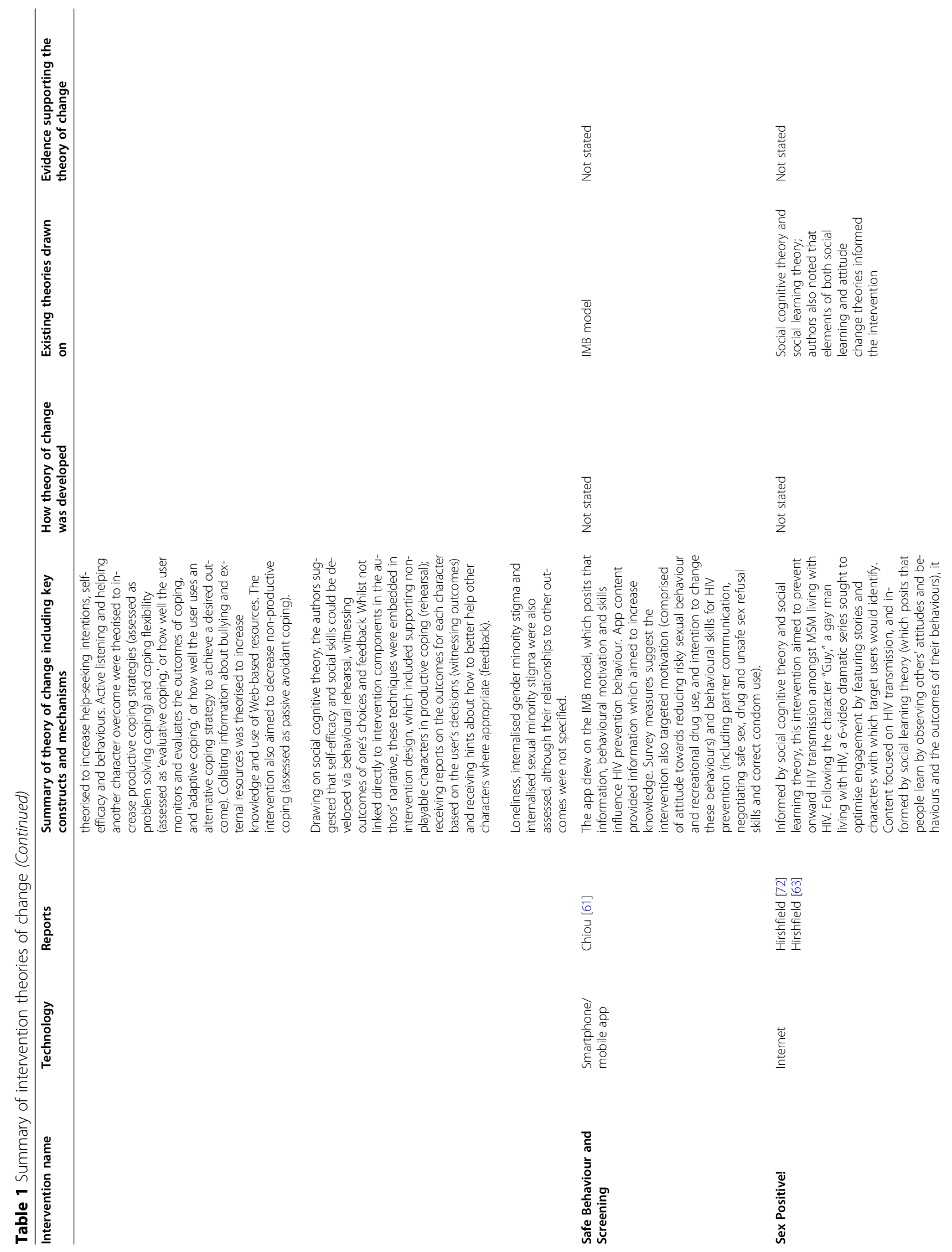




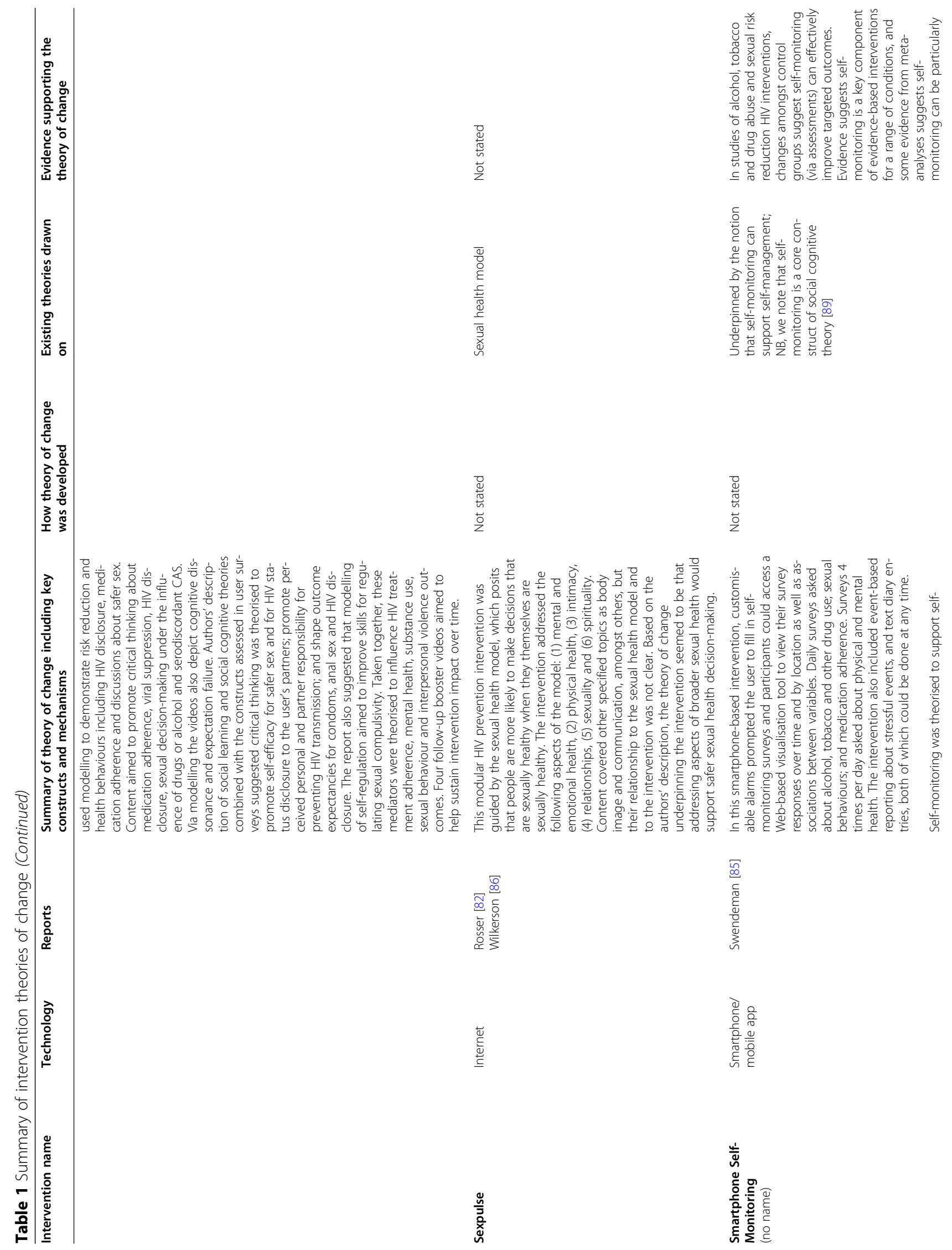




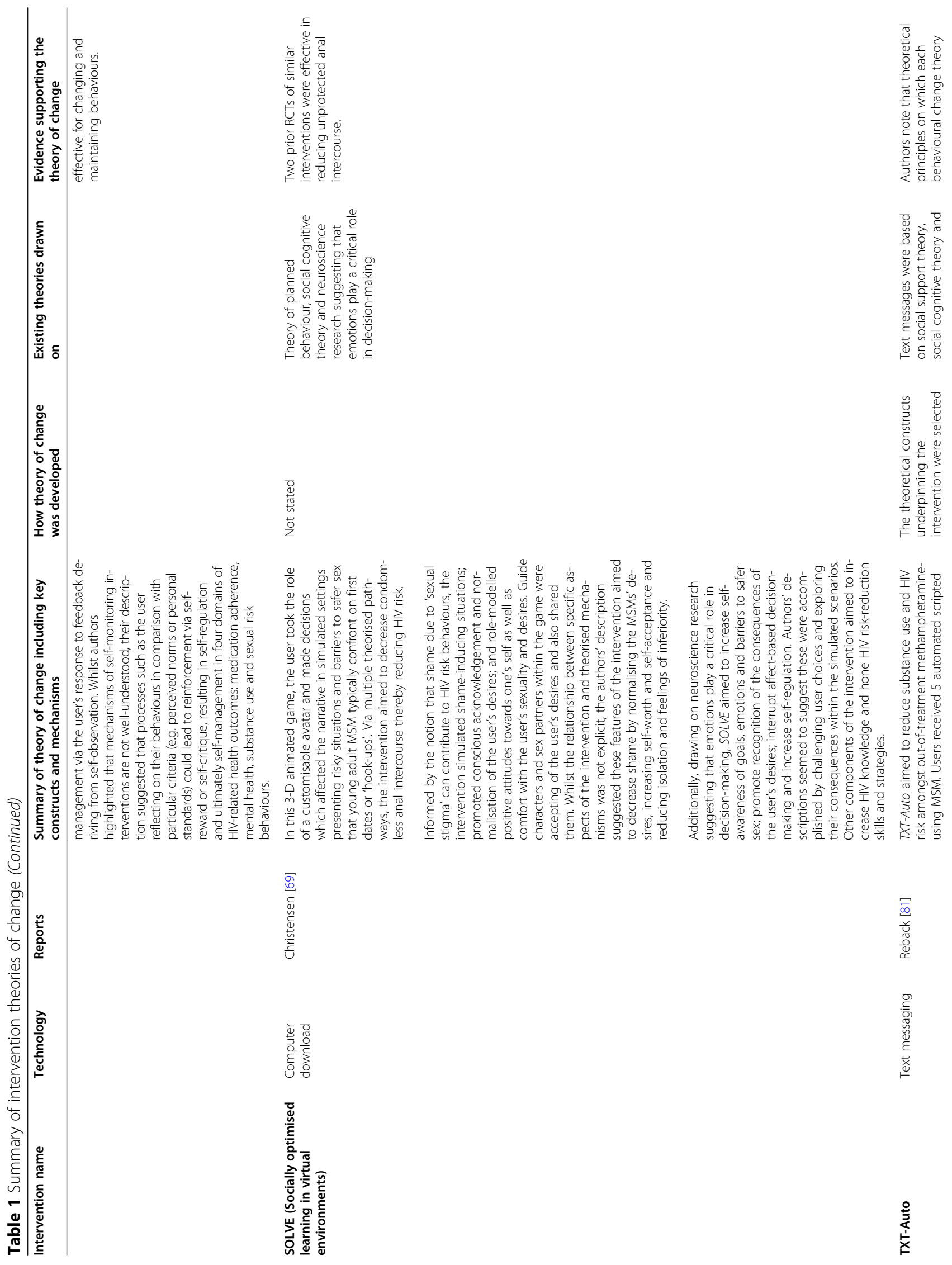




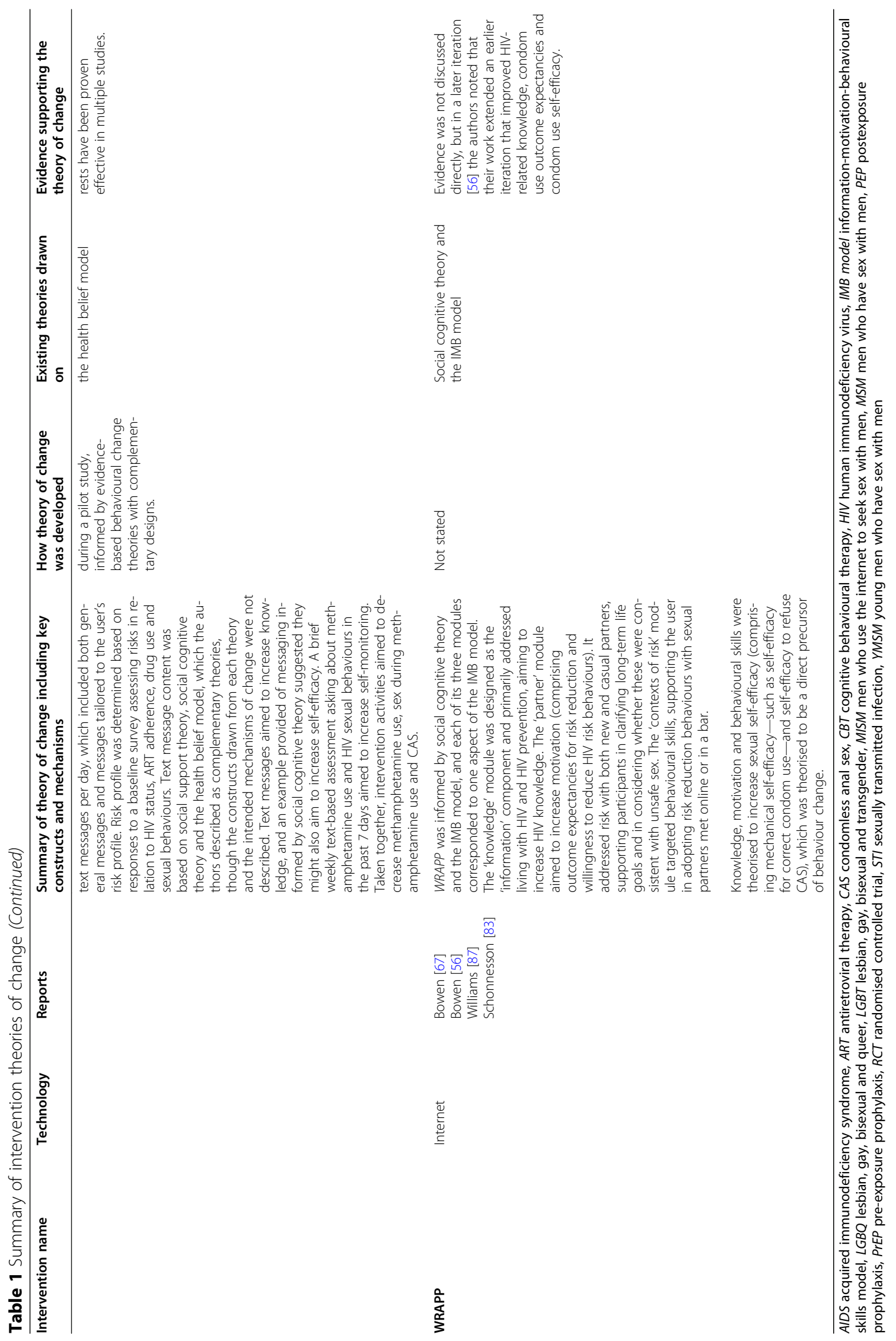




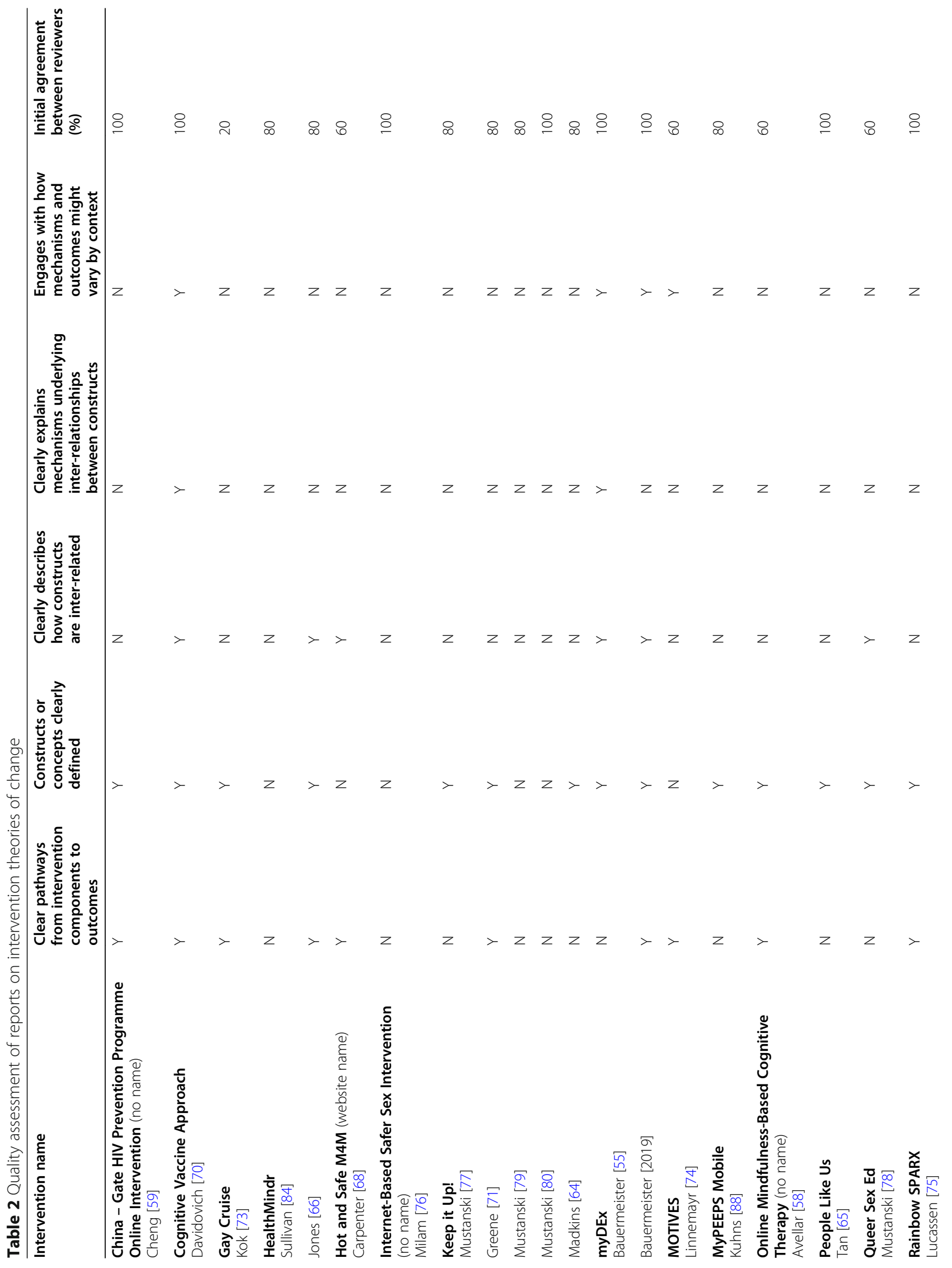




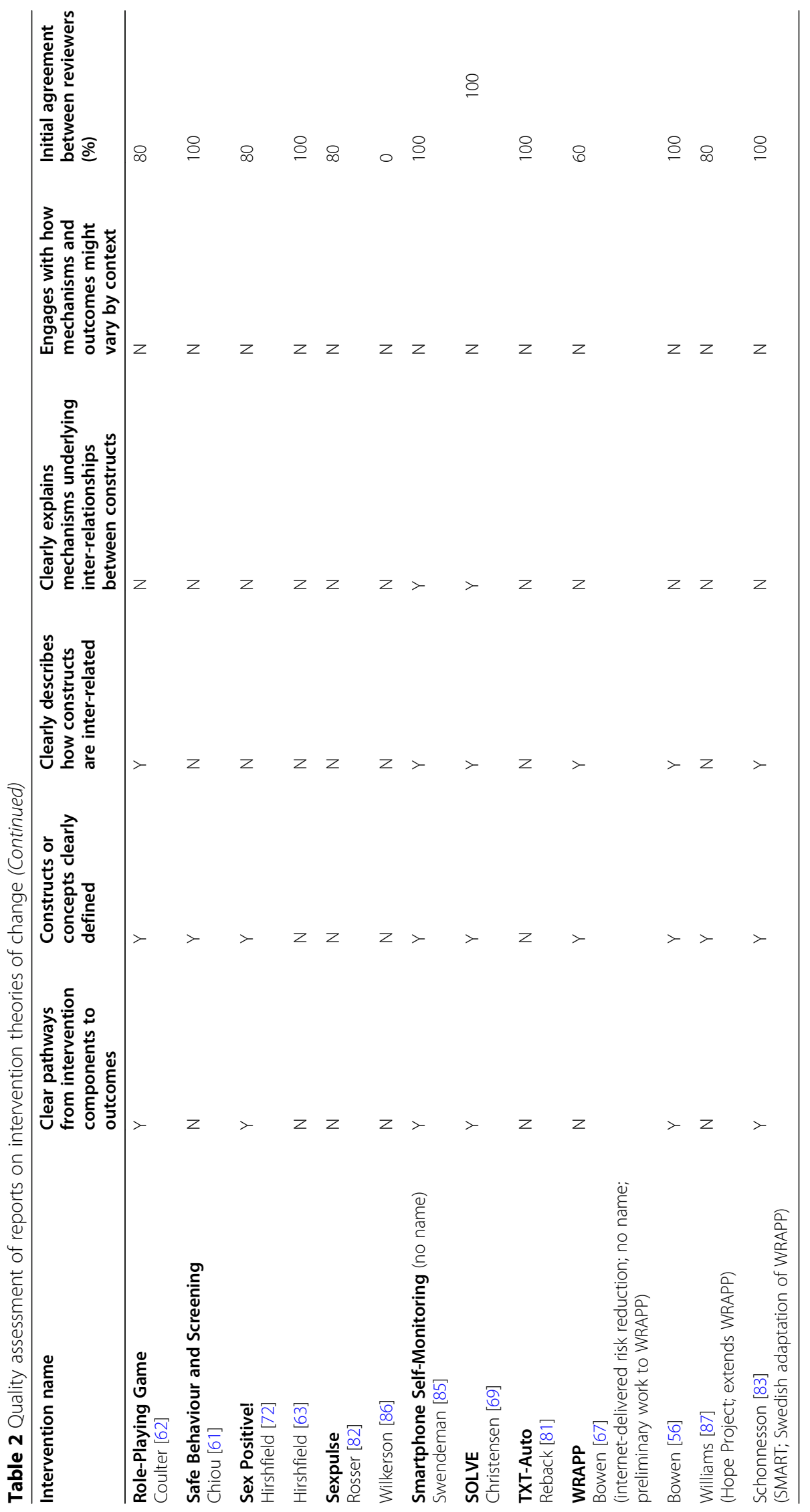


decisions' ([82], p. 2). In addition to social cognitive theory, the Role-Playing Game intervention was informed by stress and coping theory and by the emotional learning framework [62]. In regard to bullied youth, the former posits that youths' appraisals of their experience predict their coping strategies, with those who blame themselves, perceive little control and view bullying as a threat instead of a challenge tend to use non-productive coping strategies. The emotional learning framework specifies four health-promoting competencies, described as 'awareness of self and others, responsible decision making, positive attitudes and values, and social interaction skills' ([62], p. 6).

The MOTIVES intervention was rooted in behavioural economics [74], which examines how actors make decisions other than via conscious reasoning. Two interventions were underpinned by theory on interactions between cognitive and emotional factors: $m y D E x$ was informed by a 'dual processing, cognitive-emotional decision making framework' (p. 4), which recognises that affective states (emotions) and cognitive states (thinking) both influence decision-making, and that the former might be processed more quickly than the latter [55]. The SOLVE intervention was informed by learning from neuroscience highlighting the important role emotions play in decision-making [69].

Scientific theories of behaviour change were also cited, such as the transtheoretical model $[73,76]$, which maps stages and processes by which people change their behaviours [97]. The Hot and Safe M4M intervention incorporated strategies from motivational interviewing by assessing, and delivering exercises based on, participants' 'readiness to change' ([68], p. 552). Two interventions were rooted in cognitive behavioural therapy (CBT), which examines inter-relationships between cognitions and behaviours and how these may be modified: the Online Mindfulness-Based Cognitive Therapy integrated mindfulness and CBT approaches [58] and the Rainbow SPARX intervention used a computer game format to deliver CBT [75].

As evidence to support intervention components and theories of change, theory reports often cited evaluations of earlier iterations of the current intervention or of similar interventions, or previous research on the scientific theories in question. Where authors discussed how the intervention theory of change was developed, these were commonly informed by formative research, existing interventions, literature on the needs of the target group, components of the scientific theories underpinning the intervention or a combination of these.

\section{Theory of change synthesis}

All intervention theories of change identified intended intervention components, mediators and outcomes which could be incorporated into an intervention-specific theory of change diagram except for the theory underpinning the Internet-Based Safer Sex Intervention [76], which did not identify mediators. Two theories of change also identified participant characteristics theorised to moderate the relationship between the intervention and its intended outcomes $[55,60,74]$.

Our grouping of theories of change based inductively on their key constructs resulted in three groups of intervention theories of change. The largest, the 'cognitive/ skills' theory of change grouping [55, 56, 59, 61, 65, 67, $68,70-73,77-80,83,84,87,88]$, was informed largely by social cognitive theory and the IMB model. The second grouping drew on two theories of change driven primarily by self-monitoring (the 'self-monitoring' theory of change grouping) [81, 85], and the third drew on two theories of change based on cognitive therapy approaches (the 'cognitive therapy' theory of change grouping) $[58,98]$. Five theories of change did not fall within any of the three inductive groupings $[62,69,74,76,82$, 86]. The 'cognitive therapy' theory of change grouping comprised only mental health interventions. The other two theories of change groupings were not patterned by intended outcomes.

\section{'Cognitive/skills' synthesised theory of change}

We drew on thirteen intervention theories of change, which varied in quality from low to high, to develop a diagram for the 'cognitive/skills' grouping (Fig. 2) [55, $56,59-61,63-68,70-73,77-79,83,84,87,88]$. Ten of the 13 theories of change in this grouping referenced social cognitive theory $[56,63,66,67,72,73,83,84,87]$ and/or the IMB model $[55,56,60,61,64,67,68,70,71$, $77-80,83,87]$, often in combination with other scientific theories. Three referenced the theory of planned behaviour $[59,70,88]$ and one referenced no existing scientific theories but shared key constructs with other theories of change in this grouping [65]. Whilst other interventions also drew on these scientific theories, the theories of change in this grouping shared as core components constructs that are key to these three scientific theories. All interventions represented in this grouping targeted sexual health outcomes, either alone or in combination with substance use or both substance use and mental health. Two interventions not included in this grouping were primarily rooted in different approaches or scientific theories but included components both overlapping with and complementing those in this grouping [74, 76]. Using the line-of-argument approach, we drew on these to augment findings within the 'cognitive/skills' grouping and these additions (theorised moderators and the provision of information based on assessed stage-ofchange) appear in the overall diagram. 


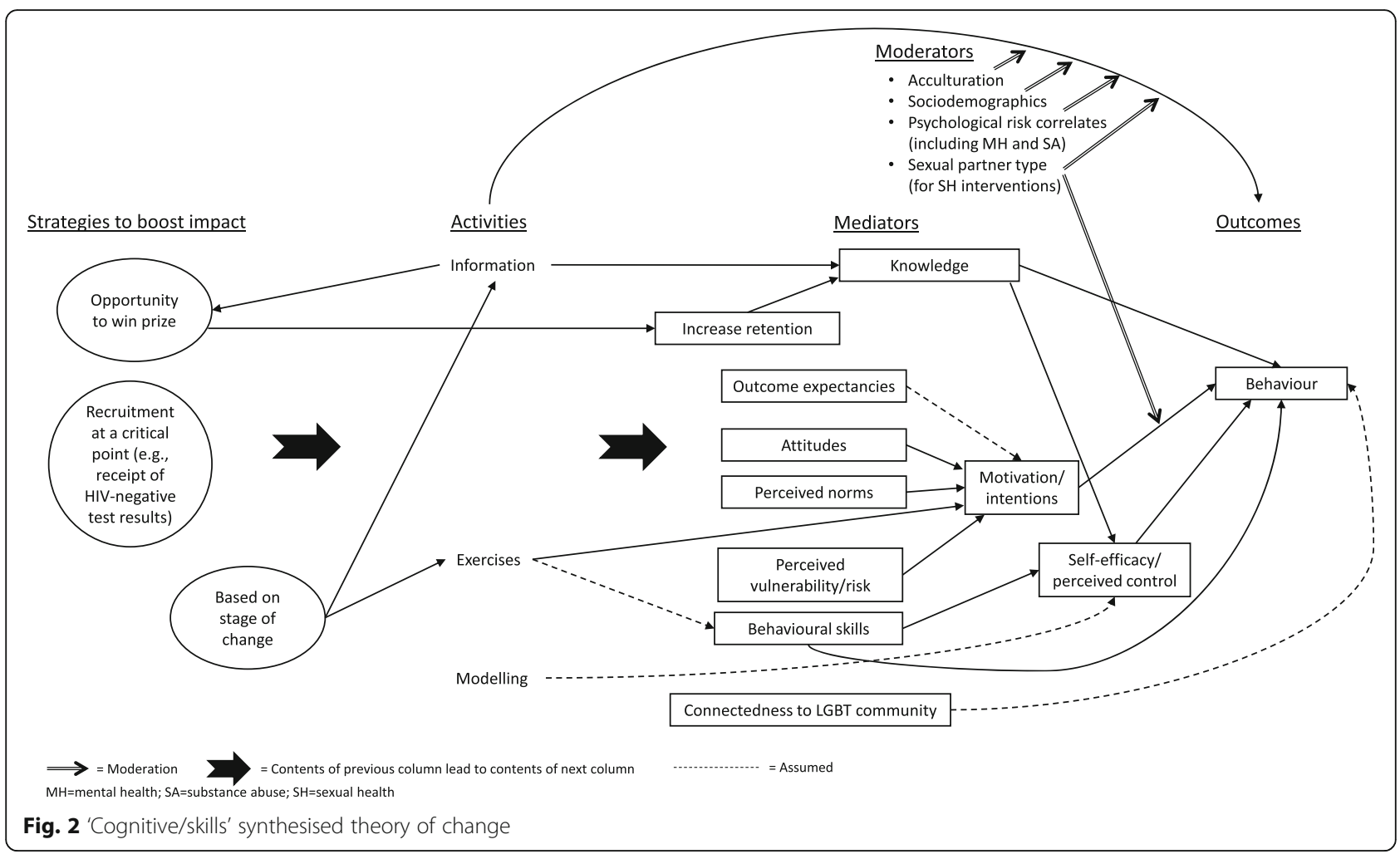

Interventions in this grouping provided information and exercises and incorporated modelling techniques. These activities were theorised to impact on mediators including knowledge, outcome expectancies, attitudes (including internalised homophobia [65, 78]), perceived norms, perceived vulnerability/risk (a construct combining via reciprocal translation those of perceived vulnerability [64, 70, 71, 77-80] and risk perceptions [59, 65, 73]) and behavioural skills. Two interventions whose theories of change informed this grouping aimed to impact connectedness to the LGBT community [65, 78], portrayed as a mediator in the synthesised diagram though the reports were not explicit about how intervention activities aimed to engender this connectedness or how it might be related to sexual health outcomes.

We inferred that exercises would develop behavioural skills, indicating this inference by use of a dotted line in Fig. 2, though behavioural skills could be developed through other activities. For example, in the video-based Sex Positive! intervention characters modelled behaviours and this was theorised to increase self-regulatory skills [72]. Theory reports did not describe clear and recurring pathways from modelling to mechanisms of change, but we inferred (denoted by use of a dotted line) from recurring descriptions that these aimed to promote self-efficacy [72, 73].

Theory reports differed as to the activities intended to influence outcome expectancies, attitudes, perceived norms and perceived vulnerability/risk. We portray the general relationship between the intervention and these mediators in the diagram by use of a block arrow. Knowledge and behavioural skills were both described as impacting behaviour either directly or via self-efficacy, so both pathways are depicted in the synthesised diagram.

Outcome expectancies-positive and negative expectations of the results of a particular behaviour [91] - were theorised as a component of motivation in the theory of change for the WRAPP intervention [56] and this was also implied in the report for the Sex Positive! intervention [72]. The theory of change for HealthMinder highlighted outcome expectations [84] as a key mediator without discussing motivation. Given these differences, we included outcome expectancies as a mediator and made the assumption that it influences motivation in the synthesised diagram.

Motivation and self-efficacy both featured prominently in this grouping's intervention theories of change. Whilst some reports suggested that motivation and self-efficacy influence each other in one direction or the other $[56,68]$, most did not address their inter-relationship, either portraying the constructs as independent $[78,79]$ or including only one [70, 72, 73, 84]. The synthesised theory of change diagram therefore portrays both constructs as impacting behaviour independently via a lineof-argument synthesis. 
Whilst some intervention theories of change included either motivation $[59,65]$ or intentions $[56,67,68,83,87]$, those that included both variably portrayed these constructs as influencing each other in one direction or the other $[55,60,78]$ or both combined into one construct $[61,70]$. We have therefore combined these constructs via reciprocal translation in the synthesised diagram.

We interpreted the construct of perceived behavioural control $[55,70]$ to be similar to the concept of selfefficacy because the latter was variously described as including an 'ability to refuse to have anal sex if a condom was unavailable' ([56], p. 7), 'confidence in practicing safer sex behaviours' ([77], p. 3003) and/or the 'ability to avoid the situational temptation to have unprotected sex' ([77], p. 3003). Self-efficacy and perceived control were therefore merged via reciprocal translation in the synthesised theory of change diagram.

Theorised moderators are shown at the top right of Fig. 2. In a few cases, theory reports discuss how these might operate $[55,74]$. Bauermeister et al. theorise that MSM's ability to enact sexual risk reduction behaviours might vary by partner type (casual encounter, romantic interest or friend with benefits) and that MSM experiencing stressors related to being a sexual minority, experiencing psychological distress or using alcohol or drugs might have less behavioural control, limiting their ability to 'regulate their affective motivations' (p. 5) and enact sexual risk reduction behaviours [55]. Linnemayr et al. theorise that participants in the MOTIVES intervention (which is not included in this grouping but augments its findings via line-of-argument synthesis) who are more acculturated to the USA and can more easily communicate with healthcare providers might face fewer barriers to HIV testing, thus benefiting less from the intervention [74].

Finally, some interventions in this grouping [68, 77] and some from outside of it [74, 76] aimed to boost intervention impact, for example, by recruiting participants at a critical point for behaviour change [77]; delivering content based on the participant's assessed stageof-change $[68,76]$; or using prize lotteries to incentivise knowledge retention [74].

\section{'Self-monitoring' synthesised theory of change}

We defined a grouping focused on theorised 'self-monitoring' (Fig. 3) by synthesising theories of change underpinning Smartphone Self-Monitoring [85], an intervention addressing all three health outcomes examined in our review, with a theory report assessed as of high quality, and the TXT-Auto intervention [81], a sexual health and substance use intervention with content tailored by the user's risk profile, with a theory of change description assessed as of medium quality. The synthesised theory of change posits that self-monitoring via frequent behavioural surveys prompts the user to reflect on their behaviour and reinforces the desired behaviour, which increases behavioural regulation and leads to behaviour change.

Both interventions in this grouping asked the user questions about their behaviour to prompt self-monitoring, and both were driven by self-monitoring as a key mechanism. Frequency of self-monitoring varied by study and outcome, ranging from four times per day [85] to weekly [81]. The theory report for Smartphone Self-Monitoring suggested the need for users to first establish criteria for their desired behaviour, such as personal norms or standards, against which they can assess their actual behaviour [85]. However, there was no evidence that this was a separate stage of the intervention so this stage does not appear in the synthesised diagram. The synthesised theory of change posits that behavioural questions result in self-monitoring, which prompts reflection in terms of pre-established criteria. This reflection is theorised to result in either self-reward or selfcritique, generating enhanced self-regulation, a theoretical mediator of behaviour change.

Whilst both theory reports included in this grouping suggest processes of change are more complex than depicted in this synthesised theory, neither specified further self-monitoring mechanisms prospectively. The theory of change underpinning TXT-Auto suggested the intervention worked via two non-intersecting pathways, one captured in the 'social/skills' synthesised theory of change and one featuring self-monitoring. Arguing that pathways from self-monitoring to behaviour change are underdeveloped, Swendeman et al.'s research drew on qualitative data to further explicate the theory of change for Smartphone Self-Monitoring [85].

\section{'Cognitive therapy' synthesised theory of change}

We drew on theories of change underpinning Online Mindfulness-Based Cognitive Therapy [58] and Rainbow SPARX [75], a computer game intervention, to develop a 'cognitive therapy' grouping (Fig. 4). Descriptions of both were assessed as of medium quality. These interventions were the only two in this review that targeted mental health outcomes alone, and both drew on cognitive therapy techniques to reduce depression and improve mental health.

Activities in the synthesised theory of change diagram include CBT approaches (forming the core of the Rainbow SPARX intervention) [75] and mindfulness-based cognitive therapy, which combines mindfulness with cognitive behavioural techniques [58]. Whilst we do not detail all activities comprising each of these approaches in the synthesised theory of change diagram, we include key components of each: behavioural training (CBT), an emphasis on prioritising 'being' over 'doing' (mindfulness-based cognitive therapy) and relaxation training (both approaches). 


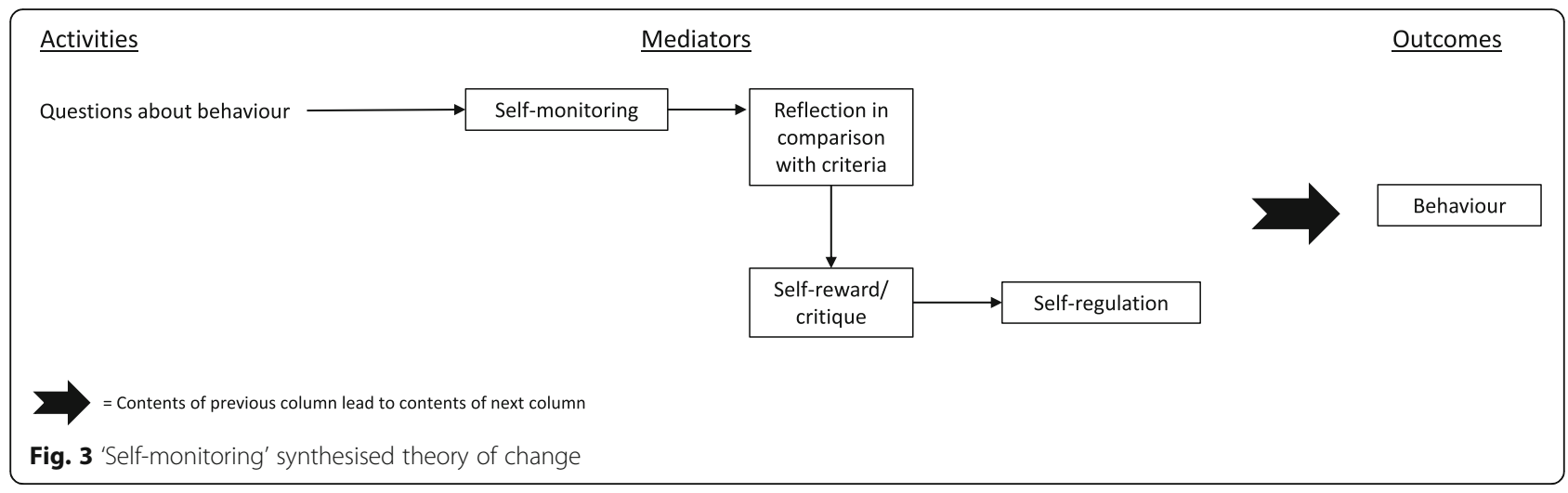

Theory of change descriptions for both interventions referred to recognising, paying attention to or developing awareness of thoughts, feelings and situations, with Rainbow SPARX [75] then challenging negative cognitions and Online Mindfulness-Based Cognitive Therapy focusing on accepting and 'letting go' of them [58]. We use differently shaded block arrows in Fig. 4 to delineate these distinct pathways. Both sought to reduce internalised homophobia and mitigate its effects on health $[58,75]$. The report for Rainbow SPARX suggested this was done by providing information to promote young people spending more time with those who accepted them and reducing exposure to homophobic bullying [75], whilst the report for Online Mindfulness-Based Cognitive Therapy did not make clear how this would be achieved [58]. Descriptions of both interventions' theories of change suggest that ultimately the reframing of distressing emotions is theorised as a key mechanism for improving mental health.

\section{Intervention theories of change not included in synthesised models}

Whilst informed by the sexual health model, the theory of change underpinning the Sexpulse intervention did not specify components or mechanisms and so could not be synthesised [82, 86]. Four other interventions, with theory reports ranging from low to high quality, did not fit within any of the three inductive theory of change groupings $[62,69,74,76]$. These interventions cut across the targeted health outcomes. Whilst some components of their theories of change overlapped with, or complemented, the above groupings, theories of change for these interventions featured elements that differentiated them from those included in the groupings. The MOTIVES [74] intervention was based on behavioural economics, and the SOLVE intervention [69] was driven by the theorised role of emotions in decision-making, featuring 'shame associated with sexual stigma' (p. 3) as a

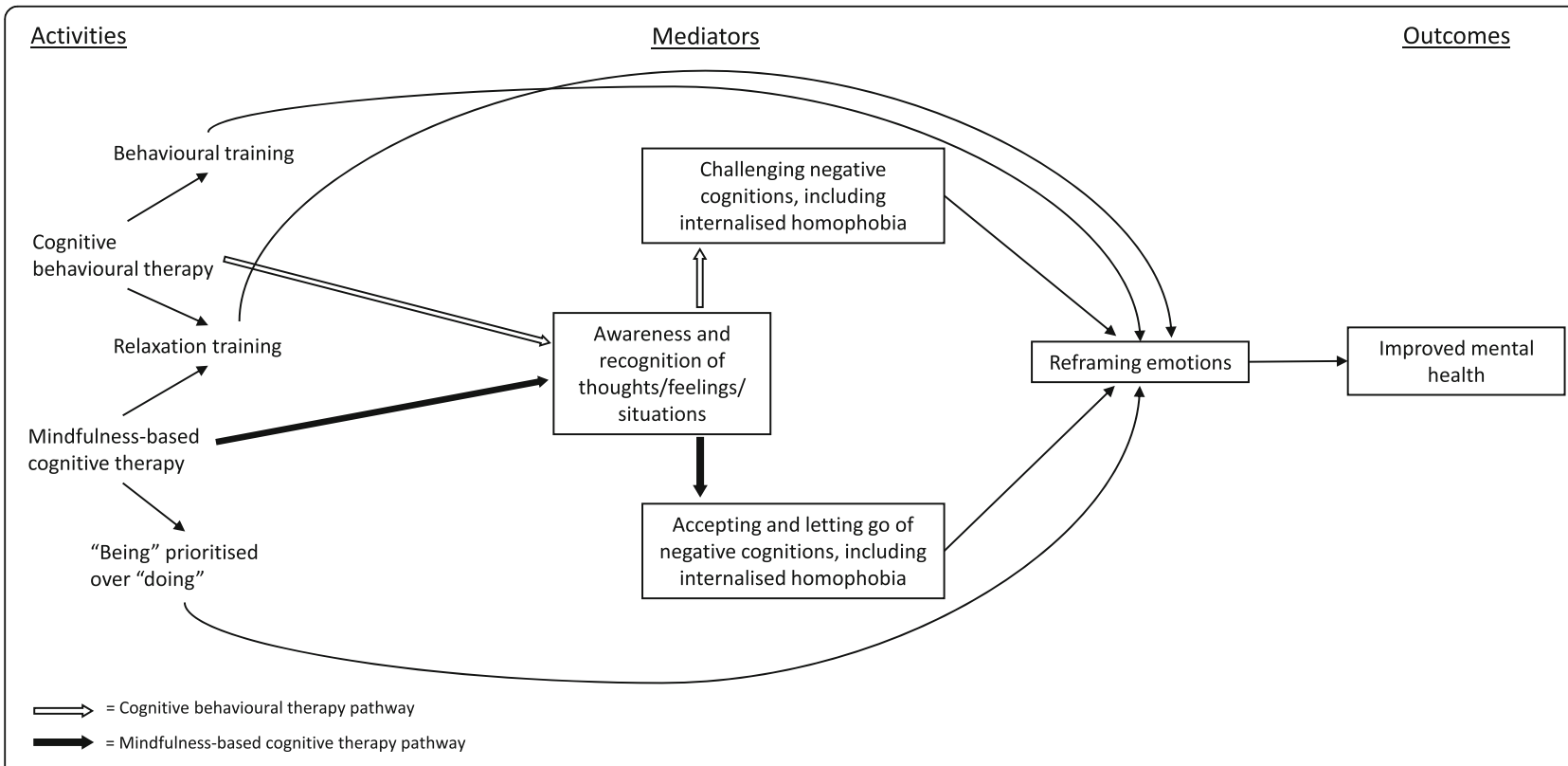

Fig. 4 'Cognitive therapy' synthesised theory of change 
key mediator. Drawing on social cognitive theory, stress and coping theory and the social and emotional learning framework, the Role-Playing Game aimed to improve mental health and reduce substance use via use of productive coping strategies with a focus on help-seeking [62]. Informed by the transtheoretical model, the theory report for the Internet-Based Safer Sex Intervention [76] suggested that the intervention was primarily based on a stages-of-change approach, tailoring content based on responses to monthly sexual behaviour surveys. The MOTIVES intervention [74] and Internet-Based Safer Sex Intervention [76] theory reports augmented the 'cognitive/skills' synthesised theory of change diagram.

\section{Discussion}

\section{Summary of findings}

For this review, we synthesised the theories of change underpinning e-health interventions targeting HIV/STIs, sexual risk behaviour, substance use and mental ill health amongst MSM. We developed a novel approach to do so by using diagrams rather than narrative themes to summarise theories of change within and across interventions.

Whilst we have previously conducted reviews synthesising intervention theories of change using line-by-line coding of descriptive text $[51,52]$, we found that this approach did not capture the often precisely described and complex inter-relationships between theoretical constructs presented in the body of literature for this review. Theories of change included in our past reviews, which addressed the integration of health and academic education [52] and positive youth development interventions [51], were relatively simple and either not significantly informed by existing scientific theories [52] or informed by theories that are not typically portrayed visually [51]. In contrast, theories of change underpinning the ehealth interventions included in this review were more complex, more explicitly theorised and largely drew on existing scientific theories which are typically conceptualised in terms of diagrams indicating relationships between their theoretical constructs, with constructs widely recognised and understood and therefore not always discussed at length. Whilst thematic analysis is a good way of rendering explicit what is implicit, it is less appropriate where the literature itself is more explicit. We therefore developed a novel method of theory of change synthesis in which we created diagrammatic summative logic models of intervention theories of change. By inductively grouping these models according to their core constructs and using meta-ethnographic approaches, we identified three emergent theoretical approaches underpinning the included interventions and we created synthesised models of each approach. We have thus synthesised theories of change underlying interventions with similar approaches. These summarise and integrate existing theories of change rather than providing a novel overarching theory of change for such interventions.

Social cognitive theory and the IMB model featured most prominently in intervention theories of change and informed a small majority. Whilst reports cited a number of other existing scientific theories, each informed only a few interventions at most. In the 'cognitive/skills' synthesised grouping, based on theory of change descriptions assessed as ranging from low to high quality, information and exercises are theorised to influence behavioural skills and a range of distal cognitive mediators theorised to influence behaviour via motivation/intention and self-efficacy/ perceived control. The synthesised theory of change suggests that particular strategies can boost intervention impact, whilst other factors relating to the participant or (for sexual health interventions) their partner moderate impact.

Though represented in fewer interventions, two other distinct theories of change groupings emerged. The 'selfmonitoring' grouping, based on theory of change descriptions assessed as of low and high quality, focuses more narrowly on the role of self-monitoring in triggering reflection, self-reward/critique and behavioural selfregulation. Theory reports suggest this synthesised diagram represents the basic components of more complex cognitive pathways [85] and can sit alongside other distinct mechanisms of behaviour change in intervention theories of change [81]. Underpinning interventions focused solely on mental health and based on theory of change descriptions assessed as of medium quality, the 'cognitive therapy' grouping is rooted in cognitive therapy techniques which can be augmented by mindfulness. In this approach, activities promote awareness and recognition of the participant's thoughts, feelings and situations and, via either challenging or accepting negative cognitions, aim to reframe negative emotions to improve mental health.

Theories of change included in this review were informed by scientific theories that have been associated with greater impacts in reviews of e-health interventions specifically, including the transtheoretical model $[41,45]$ and the theory of planned behaviour [45], though these approaches underpinned a minority of interventions. Several also featured modelling and self-monitoring, behaviour change techniques [99] which some evidence suggests might be effective in e-health interventions $[45,100]$.

As is common with e-health behavioural interventions $[45,48]$, the theories of change underpinning interventions in this review tended to rely on individually focused psychological theories of behaviour or behaviour change. Whilst these are unlikely to address structural factors contributing to the syndemic such as marginalisation, homophobia and discrimination $[19,101]$, the 
accessibility and anonymity of e-health interventions offer one approach to reducing barriers to service access stemming from stigma and discrimination [26] and might form an important element of a broader mix of interventions addressing individual and structural factors.

Furthermore, we found that included interventions drawing on multiple theoretical approaches $[55,58,65$, $69,75,78]$ took into account sexual minority-related stressors. One accounted for such stressors as a theorised moderator of intervention impact [55], whilst others aimed to increase connectedness to the LGBT community $[65,78]$ and reduce internalised homophobia $[58,65,75$, 78] and associated shame [69] to reduce sexual risk [65, $69,78]$ and improve mental health $[58,75]$. However, theory reports included little discussion of the relationships between these constructs, limiting our ability to explicate their roles in the synthesised theories of change.

It was notable that some existing scientific theories that informed theories of change were theories of behaviour change (for example, CBT theory) whilst others were theories of behaviour and its determinants (for example, the health belief model). Authors generally did not report drawing on scientific theories oriented more explicitly towards selecting strategies for enacting behaviour change, such as the Behaviour Change Wheel [102] or the Elaboration Likelihood Model [103]. Intervention developers might usefully draw on such models.

Our synthesis aimed to develop overarching theories of change for different sub-types of e-health interventions to address HIV/STIs and sexual risk, substance use and mental ill health amongst MSM, which we hope will help inform future interventions. Intervention developers might also draw on existing scientific theories which aim to integrate existing scientific theories of behaviour and behaviour change, such as the PRIME and COM-B models [102, 104].

\section{Limitations}

Our synthesis is limited by the quality of the existing theory reports, which did not often describe clear pathways from activities to intended outcomes. In some cases, reviewers inferred relationships between theory of change constructs (denoting assumptions in the theory of change diagrams), and in others the relationships between specific activities, mediators and outcomes could not be determined. Whilst our approach to theory of change synthesis enabled us to systematically explore constructs and the relationships between them across intervention theories of change, synthesised diagrams do not capture aspects that theory reports suggest influenced theories of change where their role was not clear enough to be included in intervention-specific diagrams.

We did not assess the parsimony of theories of change, because we have found in past reviews that this is very difficult to consistently operationalise as a criterion of quality assessment; however, this is an important feature of theories of change. We also did not aim to systematically assess the evidence base for each of the scientific theories underpinning the intervention theories of change, because this was outside the scope of feasibility for this review and would require assessing not only the evidence for the scientific theory but also the evidence for the application of that theory to the outcomes targeted in the theories of change it underpins.

\section{Conclusions}

e-Health interventions are a promising approach for reaching MSM with targeted health interventions [26], and existing evidence drawn from general or other populations suggests they might be effective in reducing sexual risk behaviour $[39,41]$ and substance use [30] and addressing common causes of mental ill health [31-37]. Our synthesis has identified three distinct theory of change pathways underpinning existing e-health interventions for MSM targeting sexual health, substance use and mental health outcomes, two of which underpin interventions targeting all three of these outcomes. The synthesised theories of change could provide a framework for the development of theory-driven e-health interventions holistically addressing these multiple, often co-occurring health issues amongst MSM, which can be augmented by familiarisation with the scientific theories on which they are based to inform a nuanced understanding of these theoretical underpinnings and how they can be most usefully applied in specific interventions. In the case of the 'cognitive/skills' synthesised theory of change, this would likely involve selecting a subset of mediators on which to focus, because a single intervention would not be expected to address the full range of constructs presented in this synthesised theory of change. However, our broader systematic review should first synthesise evidence on intervention effects to assess the potential of such an intervention.

Our findings suggest that the quality of existing theory reports is low- to medium, with limited discussion of the inter-relationships between theoretical constructs and little attention to how mechanisms might vary by context. Improving the quality of theory reports would enable a better understanding of how interventions are intended to work and the evidence supporting this. It would also facilitate evaluations which can go beyond outcome assessment to identify which components are triggering which mechanisms of change, and to what effect [105]. In particular, we recommend that intervention developers provide clear theories of change for their interventions, informed by existing scientific theories of behaviour and behaviour change relevant to the approach of the intervention and to the outcomes it seeks 
to address. Such theories of change can ensure intervention activities align with their intended outcomes, and can ensure that evaluations are focused on the most appropriate measures of implementation, mediators and outcomes and consider how mechanisms might vary by context and/or population.

The novel method of theory of change synthesis developed for this review worked well for the theories of change underpinning included interventions. These were largely driven by existing scientific theories and featured constructs commonly underpinning behaviour change interventions such as self-efficacy, motivation, behavioural intentions, attitudes and perceived norms. This approach can be used for similar reviews in which lineby-line coding does not effectively capture the relationships between theoretical constructs. Our forthcoming synthesis of outcome evaluations of e-health interventions addressing sexual health, substance use and/or mental health amongst MSM will aim to explore whether particular theoretical approaches are associated with greater impact.

\section{Supplementary Information}

The online version contains supplementary material available at https://doi. org/10.1186/s13643-020-01523-2.

Additional file 1. Search terms and strategy for Medline database.

Additional file 2. Individual and overarching theory of change logic models for the 'Self-monitoring' theory group.

\section{Abbreviations}

AIDS: Acquired immunodeficiency syndrome; CBT: Cognitive behavioural therapy; HIV: Human immunodeficiency virus; IMB model: Informationmotivation-behavioural skills model; MSM: Men who have sex with men; STI: Sexually transmitted infection

\section{Acknowledgements}

Not applicable

\section{Authors' contributions}

CB conceptualised and led the design of the study. AM, CW, GJMT, PW and RM contributed to the development of the study's methods. JF led the search for eligible studies. RM and CB synthesised intervention theories of change. RM led the drafting of the manuscript, with significant input from AM, CB, TCW, GJMT, JF and PW. The authors read and approved the final manuscript.

\section{Funding}

This work was funded by the National Institute for Health Research Public Health Research Programme (PHR 17/44/48). This report presents independent research commissioned by the NIHR. The views and opinions expressed by authors in this publication are those of the authors and do not necessarily reflect those of the NHS, the NIHR, MRC, CCF, NETSCC, the Public Health Research Programme or the Department of Health. The funder had no role in study design, in the collection, analysis or interpretation of data, or in writing this manuscript.

\section{Availability of data and materials}

Full details of our search strategy are available at the London School of Hygiene \& Tropical Medicine's Data Repository [50].

\section{Competing interests}

None declared.

\section{Author details}

${ }^{1}$ London School of Hygiene \& Tropical Medicine, 15-17 Tavistock Place, London WC1H 9SH, UK. ' South Cloisters, University of Exeter, St Luke's

Campus, Heavitree Road, Exeter EX1 2 LU, UK.

Received: 14 July 2020 Accepted: 8 November 2020

Published online: 11 January 2021

\section{References}

1. UNAIDS. UNAIDS data 2019. Geneva: UNAIDS, Joint United Nations, Programme on HIV/AIDS; 2019.

2. Network TE. MIS-2017 - The European Men-Who-Have-Sex-With-Men Internet Survey. Key findings from 50 countries. Stockholm: European Centre for Disease Prevention and Control; 2019.

3. Guasp A. The gay and bisexual men's health survey. London: Stonewall; 2012.

4. Buffin J. Part of the picture: lesbian, gay and bisexual people's alocohol and drug use in England (2009-2011). Manchester: The National LGB Drug \& Alcohol Database; 2012.

5. Lee JGL, Griffen GK, Melvin CL. Tobacco use among sexual minorities in the USA 1987-May 2007: a systematic review. Tobacco Control. 2009;18:275-82.

6. Vosburgh HW, Mansergh G, Sullivan PS, Purcell DW. A review of the literature on event-level substance use and sexual risk behavior among men who have sex with men. AIDS Behav. 2012;16(6):1394-410.

7. Hickson FCl, Bonell C, Weatherburn P, Reid D, Hammond G. Illicit drugs use among men who have sex with men in England and Wales. Addiction Res Theory. 2010;18(1):14-22.

8. Schmidt AJ, Bourne A, Weatherburn P, Reid D, Marcus U, Hickson F, et al. Illicit drug use among gay and bisexual men in 44 cities: findings from the European MSM Internet Survey (EMIS). Int J Drug Policy. 2016;38:4-12.

9. King M, Semlyen J, Tai SS, Killaspy H, Osborn D, Popelyuk D, et al. A systematic review of mental disorder, suicide, and deliberate self harm in lesbian, gay and bisexual people. BMC Psychiatry. 2008;8:70.

10. McFall SL. Understanding society. Colchester: Institute for Social and Economic Research, University of Essex; 2012.

11. Mercer $\mathrm{CH}$. The health and well-being of men who have sex with men (MSM) in Britain: evidence from the third National Survey of Sexual Attitudes and Lifestyles (Natsal-3). BMC Public Health. 2016;16:525.

12. Regier. Comorbidity of mental disorders with alcohol and other drug abuse. Results from the epidemiologic catchment area (ECA study). J Am Med Assoc. 1990;264(19):2511-8.

13. Colfax G, Mansergh G, Guzman R, Marks G, Rader M, Buchbinder S. Drug use and sexual risk behavior among gay and bisexual men who attend circuit parties: a venue-based comparison. JAIDS. 2001:28(4):373-9.

14. Mansergh G, Flores S, Koblin B, Hudson S, McKirnan D, Colfax GN, et al. Alcohol and drug use in the context of anal sex and other factors associated with sexually transmitted infections: results from a multi-city study of high-risk men who have sex with men in the USA. Sex Transm Infect. 2008;84(6):509-11.

15. Mustanski B, Garofalo R, Herrick A, Donenberg G. Psychosocial health problems increase risk for HIV among urban young men who have sex with men: preliminary evidence of a syndemic in need of attention. Ann Behav Med. 2007:34(1):37-45

16. Daskalopoulou M, Rodger A, Phillips AN, Sherr L, Speakman A, Collins S, et al. Recreational drug use, polydrug use, and sexual behaviour in HIVdiagnosed men who have sex with men in the UK: results from the crosssectional ASTRA study. Lancet HIV. 2014;1(1):e22-31.

17. Dearing N, Flew S. MSM: the cost of having a good time? A survey about sex, drugs and losing control; 2015 [Available from. https://doi.org/10.1136/ sextrans-2015-052126.255.

18. Heiligenberg M. Recreational drug use during sex and sexually transmitted infections among clients of a city sexually transmitted infections clinic in Amsterdam, the Netherlands. Sex Transmit Dis. 2015;39(7):518-27.

19. Stall R, Friedman M, Catania J. Interacting epidemics and gay men's health: a theory of syndemic production among urban gay men. In: Wolitski R, Stall R, Valdiserri R, editors. Unequal opportunity: health disparities affecting gay and bisexual men in the United States. New York: Oxford University Press; 2008.

20. Guadamuz T, McCarthy KW, Wimonsate W, Thienkrua W, Varangrat A, Chaikummao S, et al. Psychosocial health conditions and HIV prevalence 
and incidence in a cohort of men who have sex with men in Bangkok, Thailand: evidence of a syndemic effect. AIDS Behav. 2014;18(11):2089-96.

21. Kramer S, Schmidt A, Berg R, Furegato M, Hospers H, Folch C, et al. Factors associated with unprotected anal sex with multiple non-steady partners in the past 12 months: results from the European Men-Who-Have-Sex-WithMen Internet Survey (EMIS 2010). BMC Public Health. 2016;16(47). https:// doi.org/10.1186/s12889-016-2691-z.

22. Santos G, Do T, Beck J, Keletso M, Arreola S, Pyun T, et al. Syndemic conditions associated with increased HIV risk in a global sample of men who have sex with men. Sex Transmit Infect. 2014;90:250-3.

23. Semple S, Pitpitan E, Goodman-Meza D, Strathdee S, Chavarin C, Rangel G, et al. Correlates of condomless anal sex among men who have sex with men (MSM) in Tijuana, Mexico: The role of public sex venues. PLoS One. 2017;12(10):e0186814.

24. Ayala G, Beck J, Lauer K, Reynolds R, Sundararaj M. Social discrimination against men who have sex with men (MSM): Implications for HIV Policy and Programs. Oakland, CA; 2010 May; 2010

25. Ayala G, Makofane K, Santos G, Beck J, Do T, Hebert P, et al. Access to basic HIV-related services and PrEP acceptability among men who have sex with men worldwide: barriers, facilitators, and implications for combination prevention. J Sex Transmit Dis. 2013.

26. Ramos S, Warren R, Shedlin M, Melkus G, Kershaw T, Vorderstrasse A. A framework for using eHealth interventions to overcome medical mistrust among sexual minority men of color living with chronic conditions. Behav Med. 2019:45(2):166-76.

27. Flores J, Santos G, Makofane K, Arreola S, Ayala G. Availability and use of substance abuse treatment programs among substance-using men who have sex with men worldwide. Substance Use \& Misuse; 2017.

28. Arreola S, Santos G, Beck J, Sundararaj M, Wilson P, Herbert P, et al. Sexual stigma, criminalization, investment, and access to HIV services among men who have sex with men worldwide. AIDS Behav. 2015;19:227-34.

29. Miltz A, Rodger A, Sewell J, Speakman A, Phillips A, Sherr L, et al. 07 Depression and sexual behaviour among men who have sex with men in the UK. Sex Transmit Infect. 2015;91:A3.

30. Riper H, Blankers M, Hadiwijaya H, Cunningham J, Clarke S, Wiers R, et al. Effectiveness of guided and unguided low-intensity internet interventions for adult alcohol misuse: a meta-analysis. PLoS One. 2014;9(6):e99912.

31. Andersson G, Cuijpers P. Internet-based and other computerized psychological treatments for adult depression: a meta-analysis. Cogn Behav Ther. 2009;38(4):196-205.

32. Andrews G, Cuijpers P, Craske MG, McEvoy P, Titov N. Computer therapy for the anxiety and depressive disorders is effective, acceptable and practical health care: a meta-analysis. PLoS One. 2010;5(10):e13196.

33. Arnberg FK, Linton SJ, Hultcrantz M, Heintz E, Jonsson U. Internet-delivered psychological treatments for mood and anxiety disorders: a systematic review of their efficacy, safety, and cost-effectiveness. PLoS One. 2014;9(5):e98118.

34. Pasarelu CR, Andersson G, Bergman Nordgren L, Dobrean A. Internetdelivered transdiagnostic and tailored cognitive behavioral therapy for anxiety and depression: a systematic review and meta-analysis of randomized controlled trials. Cogn Behav Ther. 2017:46(1):1-28.

35. Spijkerman MP, Pots WT, Bohlmeijer ET. Effectiveness of online mindfulnessbased interventions in improving mental health: a review and meta-analysis of randomised controlled trials. Clin Psychol Rev. 2016;45:102-14.

36. Christensen $\mathrm{H}$, Batterham $\mathrm{P}$, Calear A. Online interventions for anxiety disorders. Curr Opin Psychiatry. 2014;27(1):7-13.

37. Kaltenthaler E, Parry G, Beverley C, Ferriter M. Computerised cognitive-behavioural therapy for depression: systematic review. Br J Psychiatry. 2008;193(3):181-4.

38. Gabarron E, Wynn R. Use of social media for sexual health promotion: a scoping review. Glob Health Action. 2016;9(1):32193.

39. Schnall R, Travers J, Rojas M, Carballo-Diéguez A. eHealth interventions for HIV prevention in high-risk men who have sex with men: a systematic review. J Med Internet Res. 2014;16(5):e134.

40. L'Engle KL, Mangone ER, Parcesepe AM, Agarwal S, Ippoliti NB. Mobile phone interventions for adolescent sexual and reproductive health: a systematic review. Pediatrics. 2016;138(3):e20160884.

41. Noar S, Black H, Pierce L. Efficacy of computer technology-based HIV prevention interventions: a meta-analysis. AIDS. 2009;23(1):107-15.

42. Rozbroj T, Lyons A, Pitts M, Mitchell A, Christensen H. Assessing the applicability of e-therapies for depression, anxiety, and other mood disorders among lesbians and gay men: analysis of 24 web- and mobile phone-based self-help interventions. J Med Internet Res. 2014;16(7):e166.
43. Sullivan P, Jones J, Kishore N, Stephenson R. The roles of technology in primary HIV prevention for men who have sex with men. Curr HIV/AIDS Rep. 2015;12(4):481-8.

44. De Silva M, Breuer E, Lee L, Asher L, Chowdhary N, Lund C, et al. Theory of change: a theory-driven approach to enhance the Medical Research Council's framework for complex interventions. Trials. 2014;15(267). https:// doi.org/10.1186/1745-6215-15-267.

45. Webb T, Joseph J, Yardley L, Michie S. Using the internet to promote health behavior change: a systematic review and meta-analysis of the impact of theoretical basis, use of behavior change techniques, and mode of delivery on efficacy. J Med Internet Res. 2010;12(1):e4.

46. Moore G, Evans R. What theory, for whom and in which context? Reflections on the application of theory in the development and evaluation of complex population health interventions. SSM Popul Health. 2017;3:132-5.

47. Daher J, Vijh R, Linthwaite B, Dave S, Kim J, Keertan D, et al. Do digital innovations for HIV and sexually transmitted infections work? Results from a systematic review (1996-2017). BMJ Open. 2017;7.

48. Nguyen L, Tran B, Rocha L, Nguyen H, Yang C, Latkin C, et al. A systematic review of eHealth interventions addressing HIV/STI prevention among men who have sex with men. AIDS Behav. 2019;23:2253-72.

49. NIHR. PROSPERO International prospective register of systematic reviews: National Institute for Health Research. 2018. [Available from: https://www. crd.york.ac.uk/prospero/display_record.php?RecordID=110317.

50. Falconer J. Search strategies for: how can e-health interventions reduce the 'syndemic' of HIV/STIs and sexual risk, substance use and mental ill health among men who have sex with men? [Data Collection]. London: London School of Hygiene \& Tropical Medicine; 2020.

51. Bonell C, Hinds K, Dickson K, Thomas J, Fletcher A, Murphy S, et al. What is positive youth development and how might it reduce substance use and violence? A systematic review and synthesis of theoretical literature. BMC Public Health. 2016;16:13.

52. Tancred T, Paparini S, Melendez-Torres G, Thomas J, Fletcher A, Campbell R, et al. A systematic review and synthesis of theories of change of school-based interventions integrating health and academic education as a novel means of preventing violence and substance use among students. Syst Rev. 2018;7(1):22.

53. Bonell C, Fletcher A, Morton M, Lorenc T. 'Realist Randomised Controlled Trials': a new approach to evaluating complex public health interventions. Soc Sci Med. 2012;75(12):8.

54. Green J, Thorogood N. Qualitative methods for health research. 4 ed. Silverman D, editor. London: Sage; 2018.

55. Bauermeister J, Tingler R, Michele Demers M, Harper G. Development of a tailored HIV prevention intervention for single young men who have sex with men who meet partners online: protocol for the myDEx Project. JMIR Res Protocol. 2017;6(7):12.

56. Bowen A, Williams M, Daniel C, Clayton S. Internet based HIV prevention research targeting rural MSM: feasibility, acceptability, and preliminary efficacy. J Behav Med. 2008;31(6):15.

57. Barnett-Page E, Thomas J. Methods for the synthesis of qualitative research: a critical review. BMC Med Res Methodol. 2009;59. https://doi.org/10.1186/ 1471-2288-9-59.

58. Avellar T. The feasibility and acceptability of an online mindfulness-based cognitive therapy intervention for same-sex attracted men: University of California Santa Barbara; 2016.

59. Cheng W, Xu H, Tang W, Zhong F, Meng G, Han Z, et al. Online HIV prevention intervention on condomless sex among men who have sex with men: a webbased randomized controlled trial. BMC Infect Dis. 2019;19(1). https://doi.org/ 10.1186/s12879-019-4251-5.

60. Bauermeister J, Tingler R, Demers M, Connochie D, Gillard G, Shaver J, et al. Acceptability and preliminary efficacy of an online HIV prevention intervention for single young men who have sex with men seeking partners online: the myDEx Project. AIDS Behavior. 2019;23(11): 3064-77.

61. Chiou P, Liao P, Liu C, Hsu Y. Effects of mobile health on HIV risk reduction for men who have sex with men. AIDS CARE. 2020;32(3):316-24.

62. Coulter R, Sang J, Louth-Marquez W, Henderson E, Espelage D, Hunter S, et al. Pilot testing the feasibility of a game intervention aimed at improving help seeking and coping among sexual and gender minority youth: protocol for a randomized controlled trial. JMIR Res Protoc. 2019;8(2).

63. Hirshfield S, Downing MJ, Chiasson M, Yoon I, Houang S, Teran R, et al. Evaluation of sex positive! A video eHealth intervention for men living with HIV. AIDS Behav. 2019;23(11):3103-18. 
64. Madkins K, Moskowitz D, Moran K, Dellucci T, Mustanski B. Measuring Acceptability and engagement of the Keep It Up! Internet-based HIV prevention randomized controlled trial for young men who have sex with men. AIDS Educ Prev. 2019;31(4):287-305.

65. Tan R, Koh W, Le D, Tan A, Tyler A, Tan C, et al. Effect of a web drama video series on HIV and other sexually transmitted infection testing among gay, bisexual and queer men: study protocol for a community-based, pragmatic randomised controlled trial in Singapore: the People Like Us (PLU) Evaluation Study. BMJ Open. 2020;10:e033855.

66. Jones J, Dominguez K, Stephenson R, Stekler J, Castel A, Mena L, et al. A theoretically based mobile app to increase pre-exposure prophylaxis uptake among men who have sex with men: protocol for a randomized controlled trial. JMIR Res Protoc. 2020;9(2):e16231.

67. Bowen A, Horvath $\mathrm{K}$, Williams M. A randomized control trial of Internetdelivered HIV prevention targeting rural MSM. Health Educ Res. 2007;22(1):8

68. Carpenter K, Stoner S, Mikko A, Dhanak L, Parsons J. Efficacy of a web-based intervention to reduce sexual risk in men who have sex with men. AIDS Behav. 2010;14(3):9.

69. Christensen J, Miller L, Appleby P, Corsbie-Massay C, Godoy C, Marsella S, et al. Reducing shame in a game that predicts HIV risk reduction for young adult men who have sex with men: a randomized trial delivered nationally over the web. J Int AIDS Soc. 2013;16:8.

70. Davidovich U, de Wit J, Stroebe W. Using the internet to reduce risk of HIVinfection in steady relationships: a randomized controlled trial of a tailored intervention for gay men; 2006.

71. Greene G, Madkins K, Andrews K, Dispenza J, Mustanski B. Implementation and evaluation of the Keep it Up! online HIV prevention intervention in a community-based setting. AIDS Educ Prev. 2016;28(3):15.

72. Hirshfield S, Downing MJ, Parsons J, Grov C, Gordon R, Houang S, et al. Developing a video-based eHealth intervention for HIV-positive gay, bisexual, and other men who have sex with men: study protocol for a randomized controlled trial. JMIR Res Protocols. 2016;5(2):14.

73. Kok G, Harterink P, Vriens P, de Zwart O, Hospers $H$. The gay cruise: developing a theory- and evidence-based internet HIV-prevention intervention. Sex Res Soci Policy. 2006;3(2):52-67.

74. Linnemayr S, MacCarthy S, Kim A, Giquere R, Carballo-Dieguez A, Barreras J. Behavioral economics-based incentives supported by mobile technology on HIV knowledge and testing frequency among Latino/a men who have sex with men and transgender women: protocol for a randomized pilot study to test intervention feasibility and acceptability. Trials. 2018;19:9.

75. Lucassen M, Merry S, Hatcher S, Frampton C. Rainbow SPARX: a novel approach to addressing depression in sexual minority youth. Cogn Behav Pract. 2015;22(2):14.

76. Milam J, Morris S, Jain S, Sun X, Dubé M, Daar E, et al. Randomized controlled trial of an internet application to reduce HIV transmission behavior among HIV infected men who have sex with men. AIDS Behav. 2016;20(6):11.

77. Mustanski B, Garofalo R, Monahan C, Gratzer B, Andrews R. Feasibility, acceptability, and preliminary efficacy of an online HIV prevention program for diverse young men who have sex with men: the Keep It Up! Intervention. AIDS Behav. 2013;17(9):14.

78. Mustanski B, Greene G, Ryan D, Whitton S. Feasibility, acceptability, and initial efficacy of an online sexual health promotion program for LGBT youth: the Queer Sex Ed Intervention. J Sex Res. 2015;42(2):11.

79. Mustanski B, Madkins K, Greene G, Parsons J, Johnson B, Sullivan P, et al. Internet-based HIV prevention with at-home sexually transmitted infection testing for young men having sex with men: study protocol of a randomized controlled trial of Keep It Up! 2.0. JMIR Res Protocols. 2017;6(1):17.

80. Mustanski B, Parsons J, Sullivan P, Madkins K, Rosenberg E, Swann G. Biomedical and behavioral outcomes of Keep It Up!: an eHealth HIV prevention program RCT. Am J Prev Med. 2018;55(2):8.

81. Reback C, Fletcher J, Swendeman D, Metzner M. Theory-based text-messaging to reduce methamphetamine use and HIV sexual risk behaviors among men who have sex with men: automated unidirectional delivery outperforms bidirectional peer interactive delivery. AIDS Behav. 2019;23(1):11

82. Rosser B, Oakes J, Konstan J, Hooper S, Horvath K, Danilenko G, et al. Reducing HIV risk behavior of MSM through persuasive computing: results of the Men's INTernet Study (MINTS-II). AIDS. 2010;24(13):9.

83. Schonnesson L, Bowen A, Williams M. Project SMART: preliminary results from a test of the efficacy of a Swedish Internet-Based HIV risk-reduction intervention for men who have sex with men. Arch Sex Behav. 2016:45(6):11.
84. Sullivan P, Driggers R, Stekler J, Siegler A, Goldenberg T, McDougal S, et al. Usability and acceptability of a mobile comprehensive HIV prevention app for men who have sex with men: a pilot study. JMIR Mhealth Uhealth. 2017;5(3):14.

85. Swendeman D, Ramanathan C, Baetscher L, Medich M, Scheffler A, Comulada W, et al. Smartphone self-monitoring to support selfmanagement among people living with HIV: perceived benefits and theory of change from a mixed-methods, randomized pilot study. J Acquir Immune Defic Syndr. 2015;69:12.

86. Wilkerson J, Danilenko G, Myer B, Rosser B. The role of critical self-reflection on assumptions in changes in sexual beliefs and behaviors by men who use the internet to seek sex with men. AIDS Educ Prev. 2011;23(1):12.

87. Williams M, Bowen A, Ei S. An evaluation of the experiences of rural MSM who accessed an online HIV/AIDS health promotion intervention. Health Promot Pract. 2010;11(4):9.

88. Kuhns L, Garofalo R, Hidalgo M, Hirshfield S, Pearson C, Bruce J, et al. A randomized controlled efficacy trial of an mHealth HIV prevention intervention for sexual minority young men: MyPEEPS mobile study protocol. BMC Public Health. 2020;20(65). https://doi.org/10.1186/s12889-020-8180-4.

89. Bandura A. Social cognitive theory of self-regulation. Org Behav Hum Decision Processes. 1991;50(2):40.

90. Fisher W, Fisher J, Harman J. The information-motivation-behavioral skills model: a general social psychological approach to understanding and promoting health behavior. In: Suls J, Wallston K, editors. Social Psychological Foundations of Health and IIness. Blackwell Series in Health Psychology and Behavioral Medicine. Malden: Blackwell Publishing Ltd; 2009.

91. Bandura A. Health promotion from the perspective of social cognitive theory. Psychol Health. 1998;13:27.

92. Bozack A. Social cognitive learning theory. In: Springer, editor. Encyclopedia of Child Behavior and DevelopmentEncyclopedia of Child Behavior and Development. Boston: Apringer; 2011.

93. Becker M. The health belief model: a decade later. Health Educ Behav. 1984; 11(1):1-47.

94. Ajzen I. The theory of planned behavior. Organizational behavior and human decision processes. 1991;50(2):179-211.

95. Reback C, Fletcher J, Shoptaw S, Mansergh G. Exposure to theory-driven text messages is associated with HIV risk reduction among methamphetamineusing men who have sex with men. AIDS Behav. 2015;19:130-41.

96. Robinson B, Bockting W, Rosser B, Miner M, Coleman E. The sexual health model: application of a sexological approach to HIV prevention. Health Educ Res. 2002;17(1):43-57.

97. Prochaska J, Redding C, Evers K. The transtheoretical model and stages of change. In: Glanz K, Rimer B, Viswanath K, editors. Health Behavior: Theory, Research, and Practice. San Francisco: Jossey-Bass; 2015.

98. Lucassen M, Hatcher S, Fleming T, Stasiak K, Shepherd M, Merry S. A qualitative study of sexual minority young people's experiences of computerised therapy for depression. Aust Psychiatr. 2015;23(3):6.

99. Abraham C, Michie S. A taxonomy of behavior change techniques used in interventions. Health Psychol. 2008;27(3):379-87.

100. Morrison L, Yardley L, Powell J, Michie S. What design features are used in effective eHealth interventions? A review using techniques from critical interpretative synthesis. Telemed e-Health. 2012;18(2):137-44.

101. Halkitis P. Discrimination and homophobia fuel the HIV epidemic in gay and bisexual men. Psychol AIDS Exchange Newsletter. April 2012.

102. Michie S, van Stralen M, West R. The behaviour change wheel: a new method for characterising and designing behaviour change interventions. Implement Sci. 2011;6(42). https://doi.org/10.1186/1748-5908-6-42.

103. Petty R, Cacioppo J. The elaboration likelihood model of persuasion. Adv Exp Soc Psychol. 1986;19:123-205.

104. West R. The PRIME theory of motivation as a possible foundation for addiction treatment. In: Henningfield J, Santora P, editors. Drug Addiction Treatment in the 21st Century: Science and Policy Issues. Baltimore: Johns Hopkins University Press; 2007.

105. Moore G, Audrey S, Barker M, Bond L, Bonell C, Hardeman W, et al. Process evaluation of complex interventions: Medical Research Council guidance. BMJ. 2015;350:h1258.

\section{Publisher's Note}

Springer Nature remains neutral with regard to jurisdictional claims in published maps and institutional affiliations. 